\title{
Article
}

\section{Space-Time Quantum Imaging}

\author{
Ronald E. Meyers* and Keith S. Deacon \\ US Army Research Laboratory, 2800 Powder Mill Road, Adelphi, MD 20783, USA; \\ E-Mail: keith.s.deacon.civ@mail.mil
}

* Author to whom correspondence should be addressed; E-Mail: ronald.e.meyers6.civ@mail.mil; Tel: +1-301-394-2111; Fax: +1-301-394-2017.

Academic Editor: Hiromi Saida

Received: 1 October 2014 / Accepted: 27 February 2015 / Published: 23 March 2015

\begin{abstract}
We report on an experimental and theoretical investigation of quantum imaging where the images are stored in both space and time. Ghost images of remote objects are produced with either one or two beams of chaotic laser light generated by a rotating ground glass and two sensors measuring the reference field and bucket field at different space-time points. We further observe that the ghost images translate depending on the time delay between the sensor measurements. The ghost imaging experiments are performed both with and without turbulence. A discussion of the physics of the space-time imaging is presented in terms of quantum nonlocal two-photon analysis to support the experimental results. The theoretical model includes certain phase factors of the rotating ground glass. These experiments demonstrated a means to investigate the time and space aspects of ghost imaging and showed that ghost imaging contains more information per measured photon than was previously recognized where multiple ghost images are stored within the same ghost imaging data sets. This suggests new pathways to explore quantum information stored not only in multi-photon coincidence information but also in time delayed multi-photon interference. The research is applicable to making enhanced space-time quantum images and videos of moving objects where the images are stored in both space and time.
\end{abstract}

Keywords: quantum; space-time; ghost imaging; two-photon interference; ground glass; turbulence; turbulence-free; wavefunction; probability amplitude 


\section{Introduction}

Meyers et al. [1,2] in 2007 and 2008 pioneered the first ghost image of a remote object by imaging a small toy soldier in their setup at the U.S. Army Research Laboratory.

This demonstration was the first practical application of ghost imaging and it showed that ghost imaging could be applied to remote sensing from astrophysical to microscopic scales with the potential of increased resolution, increased contrast, and mitigation of adverse effects from distorting media. Other interesting Ghost imaging research includes experiments on entangled photon ghost imaging through laboratory turbulence [3], signal-to-noise studies and illumination variations [4,5], studies on contrast and visibility [6,7], virtual or computational ghost imaging [8-10], along with associated fundamental experimental [11] and theoretical physics [12-14]. Extending the remote ghost imaging practical application to a turbulent environment, Turbulence-free Ghost Imaging (Meyers et al. $[15,16]$ ) was recently proven, wherein turbulence has virtually no adverse effect on ghost imaging as shown in Figure 1.
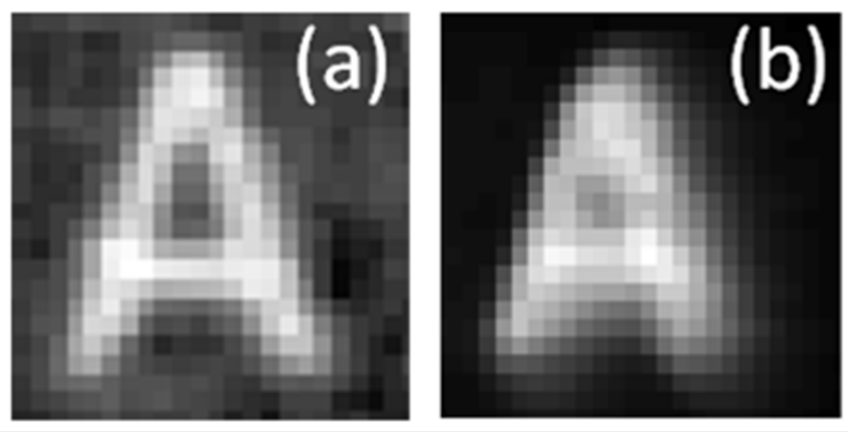

Figure 1. Ghost imaging through turbulence results: (a) a turbulence-free ghost image of the letter "A", (b) a classical averaged image of the letter "A". Meyers, et al. [15]

Although the theory interpreting these ghost imaging experiments evolved from a more general approach where photons are emitted at two separate space-time points, the measurements presented in the ghost images were time coincident. In particular we asked the question, "Can ghost images be generated when the two sensors of ghost imaging make photon measurements at different times?" In this paper we present our experimental findings demonstrating that new types of space-time ghost images can be generated when the measurements of the two-photon system are separated in time as well as space. In particular we show that ghost images of remote objects can be produced with either one or two beams of chaotic laser light and two sensors measuring at different space-time points.

\section{Experimental Setup}

To investigate space-time ghost imaging we needed to adjust previous experiments to examine the effect of the scattering phenomena involved in pseudo-thermal light on ghost imaging by adding measurements that resolve the two-photon coherence time of the system. The setup to perform the experiments is shown in Figure 2. It is similar to previous ghost imaging setups [1,2,15-19]. The setup differs in that sometimes a faster or slower charged coupled device (CCD) framing rate was implemented and sometimes a faster or slower ground glass rotation rate was used. A variety of space-time ghost 
imaging experiments were performed with combinations of rotating ground glass rates and CCD timing parameters. The rotation rate of the ground glass ranged from less than $1^{\circ}$ per second to greater than 1500 $\mathrm{rpm}$. The CCD timing parameters included frame rates of less than 1 frames per second (fps) to more than 1000 fps and the integration times ranged from micro-seconds to nanoseconds with some timing controls operating in picoseconds. For example at the low end of the experiments CCD integration times of $1 \mathrm{~ms}$, frame rates of approximately $13 \mathrm{fps}$, and ground glass rotation speeds as low as $1^{\circ}$ per second were implemented. We performed these experiments for successive imaging frames so as to track the laser illumination rotating ground glass disturbances over the reference and target fields. The space-time ghost imaging experiments consisted of using the reference field images at each time $t_{r e f}=t_{i}$ and a bucket field measured at a separate time $t_{b}=t_{i+\Delta i}$ where $t_{i}$ is the time of the $i^{t h}$ measurement. This was performed for each time separation of $\Delta i=-N$ to $+N$, where in some of our experiments $N=20$. We also evaluated the time correlations in the CCD frames. The experimental results showed that it was possible to resolve a ghost image with time separations up to twenty frames, that is $\Delta i= \pm 20$. The ghost image of the "ARL" moved to the left or right of the $\Delta i=0$ time separation ghost image depending on whether the reference measurement was correlated with an earlier $\left(t_{r e f}-t_{b}<0\right)$ or later $\left(t_{r e f}-t_{b}>0\right)$ "bucket" measurement as depicted at the top of Figure 2.

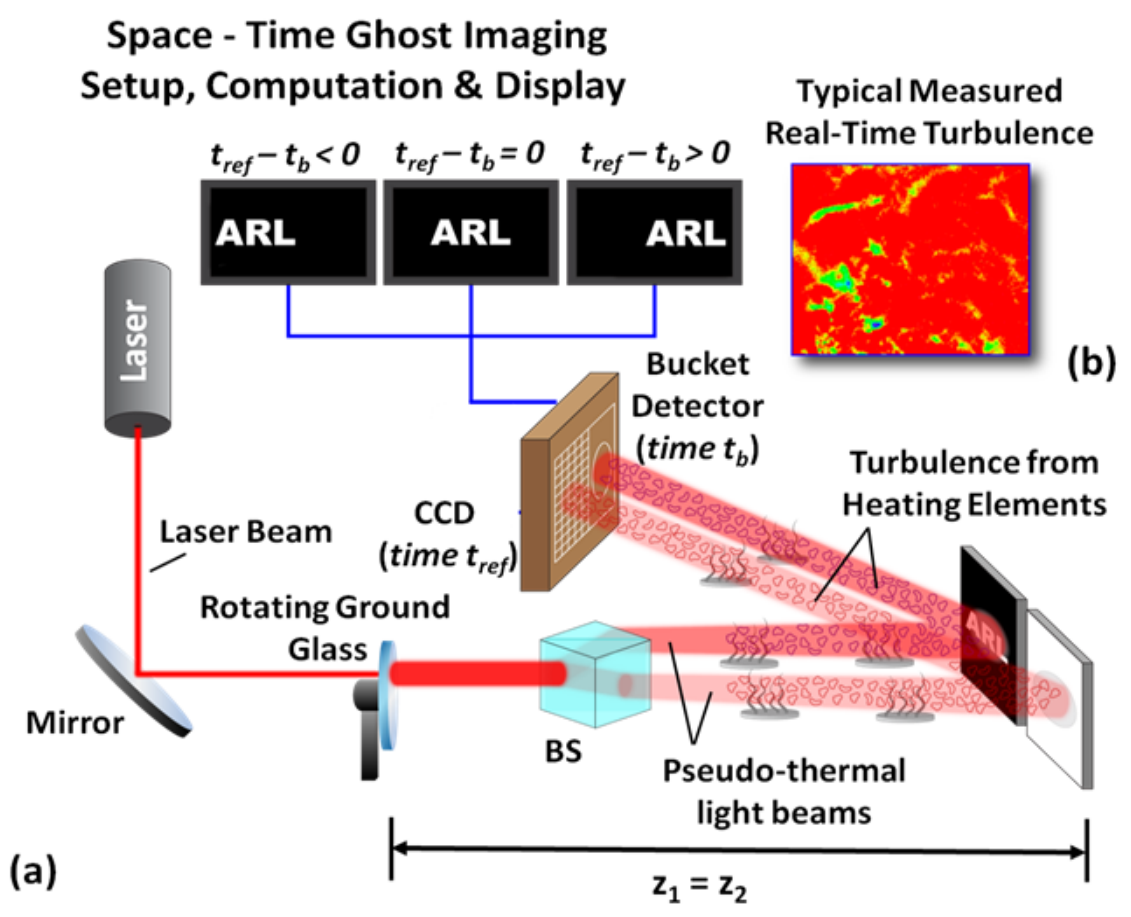

Figure 2. Layout for the space-time quantum ghost imaging experiments. A charged coupled device (CCD) collected reference field images at each time $t_{r e f}=t_{i}$ and a single pixel photo-sensor measured the bucket field at a separate time $t_{b}=t_{i+\Delta i}$, where $t_{i}$ is the time of the $i^{t h}$ measurement. The space-time "ARL" images at the top of the figure are representative results when the reference field measurements are correlated with earlier $\left(t_{r e f}-t_{b}<0\right)$, later $\left(t_{r e f}-t_{b}>0\right)$, or coincident $\left(t_{r e f}-t_{b}=0\right)$ bucket photon measurements.

These experiments were performed with and without turbulence and used a typical thermal light lensless ghost imaging setup [1]. The CCD records the secondary image of the primary ghost 
image $[15,17]$ at the reference-image arm which helps make ghost imaging practical for applications. In this experiment, atmospheric turbulence is introduced to the optical paths by the adding heating elements underneath the optical paths operating at a temperature of $550{ }^{\circ} \mathrm{C}$ with the refractive index structure parameter in the range $C_{n}^{2} \sim 1.5 \cdot 10^{-12}$ to $10^{-9}$. Note that the illumination and imaging paths traversed differing turbulence realizations each with these high levels of turbulence. In Figure 2, we illustrate the most serious situation in which turbulence occurs in all optical paths of the setup. Heating of the air causes temporal and spatial fluctuations on its index of refraction that makes the classical image of the object jitter about randomly on the image plane causing a "blurred" picture. Similar to our early experiment [1], the light source is a typical chaotic pseudo-thermal source, which contains a laser beam and a rotating ground glass diffuser. The thermalized chaotically scattered laser beam, which has a fairly large size on the ground glass $(11 \mathrm{~mm}$ diameter) in transverse dimension, is split into two by a $50 \%-50 \%$ beamsplitter (BS). One of the beams illuminates an object located at $z_{1}$, such as the letters "ARL" as shown in Figure 2. The scattered and reflected photons from the object are collected and counted by a "bucket" detector, which is simulated by the right-half of the CCD in Figure 2. The other beam propagates to the ghost image plane of $z_{2}=z_{1} \simeq 1.4 \mathrm{~m}$ and the distance from the target to the detectors is $\sim 1.7 \mathrm{~m}$. Placing a CCD array on the ghost image plane, the CCD array will capture the ghost image of the object if its exposure is gated by the bucket detector [1]. In this experiment the CCD array will image the target and reference planes located on a sheet of paper. The CCD is moved to a distance to view the ghost image on the glossy white paper. The scattered and reflected light from the glossy white half of the paper, which contains the spatial information for the ghost image, is then captured by the left-half of the high resolution CCD camera operating in the photon counting regime. The CCD camera is focused onto the glossy white paper at the ghost image plane and is gated by the bucket detector for the observation of the secondary ghost image. The hardware circuit and the software program are designed to monitor the outputs of the left-half and the right-half of the CCD individually, as two independent classical cameras, and simultaneously to monitor the gated output of the left-half of the CCD as a ghost camera. In the measurement, the classical image and the secondary ghost image of the object were captured and monitored simultaneously. In addition, measurements were made of the $g_{12 \text { deviations }}^{(2)}$ correlations between intensity deviations (fluctuations) measured at each pixel by detectors 1 and 2 and at each time which can be described by

$$
g_{12 \text { deviations }}^{(2)}=\left\langle\Delta I_{i}^{1} \Delta I_{i+\Delta i}^{2}\right\rangle=\left\langle\left[I_{i}^{1}-\left\langle I_{i}^{1}\right\rangle\right]\left[I_{i+\Delta i}^{2}-\left\langle I_{i+\Delta i}^{2}\right\rangle\right]\right\rangle
$$

where the $I_{i}^{1}, I_{i}^{2}$ are the measured photon counts at frame $i$, and $\Delta i$ is the separation from frame $i$. This is essentially the space-time correlation of intensity deviations (fluctuations). Also, the normalized correlation $R_{\Delta i}^{12}$ as a function of $\Delta i$ is given by

$$
R_{\Delta i}^{12}=\frac{\left\langle\left[I_{i}^{1}-\left\langle I_{i}^{1}\right\rangle\right]\left[I_{i+\Delta i}^{2}-\left\langle I_{i+\Delta i}^{2}\right\rangle\right]\right\rangle}{\sigma_{i}^{1} \sigma_{i+\Delta i}^{2}},
$$

where $\sigma_{i}^{1}$ and $\sigma_{i}^{2}$ are the standard deviations of $I_{i}^{1}$ and $I_{i}^{2}$. Equation (2) above is normalized by the standard deviations at each space-time coordinate and represents the normalized space-time correlation of intensity deviations (fluctuations). 


\section{Space-Time Ghost Imaging}

The physics of the space-time ghost imaging follows the formulation presented by Meyers et al. [1,2] for remote ghost imaging wherein the Glauber theory [20,21] was adapted to our two-photon interference ghost imaging setup.

$$
G^{(2)}\left(\mathbf{r}_{1}, t_{1} ; \mathbf{r}_{2}, t_{2}\right)=\left|\left\langle 0\left|\widehat{E}_{1}^{(-)} \widehat{E}_{2}^{(-)} \widehat{E}_{2}^{(+)} \widehat{E}_{1}^{(+)}\right| \Psi\right\rangle\right|^{2}
$$

and

$$
G^{(2)}\left(\mathbf{r}_{1}, t_{1} ; \mathbf{r}_{2}, t_{2}\right)=\left|\Psi\left(\vec{\rho}_{1}, z_{1}, t_{1} ; \vec{\rho}_{2}, z_{2}, t_{2}\right)\right|^{2}
$$

where the negative and positive frequency fields $\widehat{E}_{1}^{(-)}, \widehat{E}_{2}^{(-)}, \widehat{E}_{2}^{(+)}, \widehat{E}_{1}^{(+)}$contribute at the space-time points $\mathbf{r}_{1}, t_{1}, \mathbf{r}_{2}, t_{2}$. The wavefunction $\Psi\left(\vec{\rho}_{1}, z_{1}, t_{1} ; \vec{\rho}_{2}, z_{2}, t_{2}\right)$ is the effective two-photon wavefunction (probability amplitude). Figure 3 shows bucket and reference detectors when photons are emitted by two sub-sources. Figure 4 presents a depiction of conventional turbulence-free ghost imaging where there is a phase disturbance in the photon paths such as caused by turbulence. In that case phase disturbances cancel in the $G^{(2)}$ due to symmetry in the wavefunction.

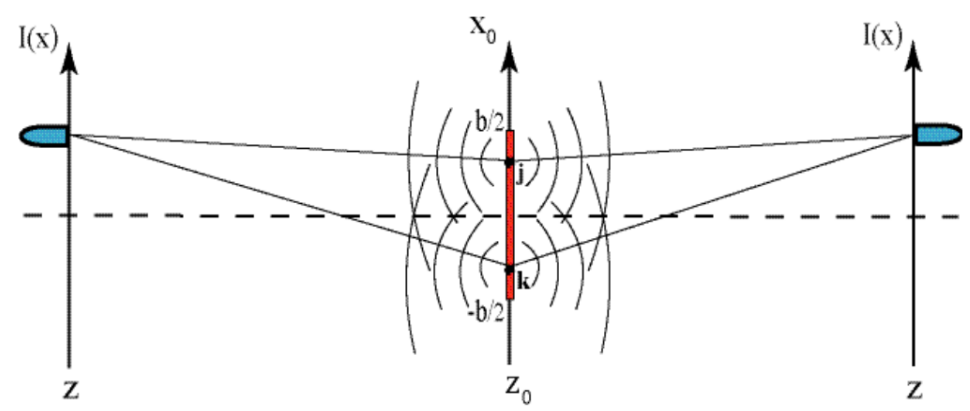

Figure 3. Typical ghost imaging representation where there are two alternate and indistinguishable ways to generate a joint-detection. Space-time quantum imaging generalizes this representation according to $G^{(2)}\left(\mathbf{r}_{1}, t_{1} ; \mathbf{r}_{2}, t_{2}\right)$.

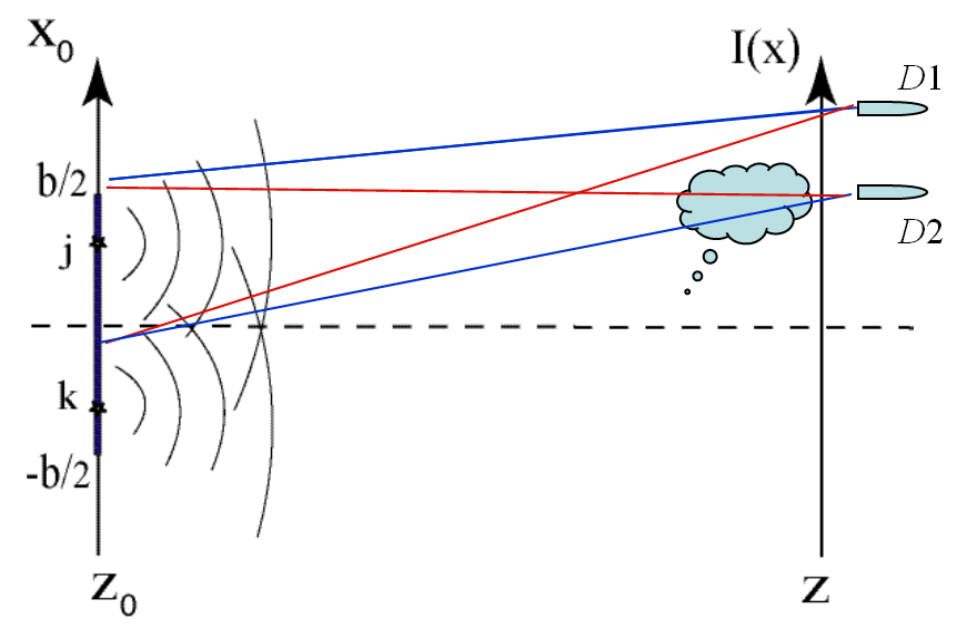

Figure 4. Representation of two-photon detection through turbulence depicting two alternate but indistinguishable ways to produce a joint detection in the presence of turbulence [15]. 


\subsection{Double Beam Results}

The ghost images depicted in this section were measured in the setup given by Figure 2 . We found that each ghost image was translated according to the value of the time separation index $\Delta i$. When played as a movie the ghost image is seen to smoothly translate across the computer screen, the amount of translation being proportional to the time separation index. The translation appeared to be consistent with the motion of the ground glass scattering centers, although the reference frame correlations were reduced to 0.023 after three frames and 0.003 after 20 frames. At first, space-time ghost imaging was performed in non-turbulent conditions where the heating elements in Figure 2 were turned off. Figure 5a1,a2 presents the results of a ghost image generated with the time separation index $\Delta i=0$. This image is typical of ghost imaging where the ghost image is produced from coincident measurements. Our next result, Figure 5b1,b2, presents ghost images of the "ARL" target produced when the time separation index $\Delta i= \pm 5$. Note that the images of the "ARL" target are translated towards the left and right relative to the $\Delta i=0$ ghost image. These ghost images of the "ARL" target are visually slightly less distinct when compared with the "ARL" ghost image computed with $\Delta i=0$.

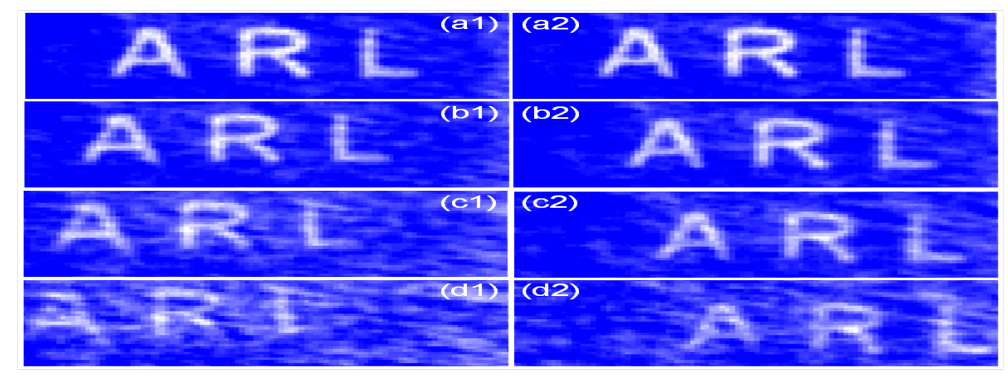

Figure 5. Correlation ghost images taken under conditions of no turbulence. (a1, a2) zero frame separation correlation ghost image. (b1) -5 frame separation correlation ghost image. (b2) +5 frame separation correlation ghost image.

We also present in Figure 6 composite results from multiple $\Delta i$ ghost images. The left side shows the composite image produced from the sum ghost images of $\Delta i=0, \pm 5, \pm 10, \pm 15$ while the right side shows the composite sum ghost image for all of the $\Delta i$ considered in this experiment (41 images).
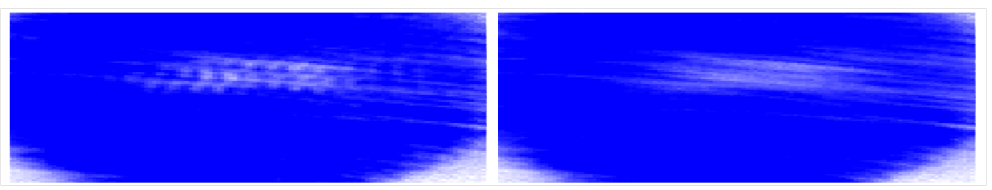

Figure 6. Left panel: Composite Ghost Image from adding $\pm 15, \pm 10, \pm 5$, and 0 time separation correlation images; Right panel: Composite Ghost Image from adding all time separation \pm 20 correlation images (41 images). No turbulence.

Next, we present the results of space-time ghost images generated with the heating elements turned on. These results are shown in Figure 7. Note again that the images in Figure 7b1,b2 of the "ARL" target are translated towards the left and right relative to the $\Delta i=0$ ghost image. Also, these ghost images through turbulence of the "ARL" target are visually quite similar with the "ARL" ghost images 
in non-turbulent conditions. In addition, measurements were made of the correlations between intensities measured at each pixel which can be described by

$$
R_{\Delta i}=\frac{\left\langle\left[I_{i}-\left\langle I_{i}\right\rangle\right]\left[I_{i+\Delta i}-\left\langle I_{i+\Delta i}\right\rangle\right]\right\rangle}{\sigma_{i} \sigma_{i+\Delta i}},
$$

where the $I_{i}$ are the measured photon counts at frame $i, \Delta i$ is the separation from frame $i$, and $\sigma_{i}$ is the standard deviation of $I_{i}$. A table of reference field intensity correlations as a function of frame time separation for the non-turbulent experiment case is shown in Table 1.

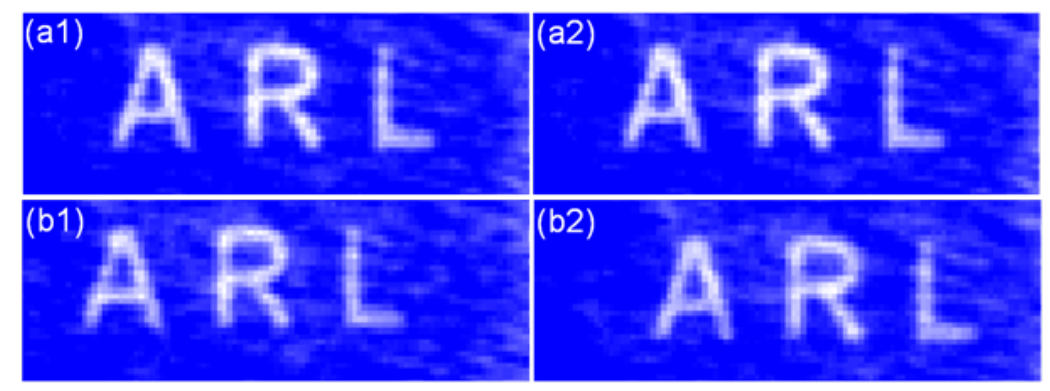

Figure 7. Correlation ghost images taken under conditions of turbulence. (a1, a2) zero frame separation correlation ghost image. (b1) -5 frame separation correlation ghost image. (b2) +5 frame separation correlation ghost image.

Table 1. Non-Turbulent Case: Reference Field Intensity Correlations as a function of time separation.

\begin{tabular}{lccccccc}
\hline$\Delta i$ & 0 & 1 & 2 & 3 & 5 & 10 & 20 \\
$R_{+\Delta i}$ & 1.0 & 0.47 & 0.09 & 0.023 & 0.009 & 0.006 & 0.003 \\
$R_{-\Delta i}$ & 1.0 & 0.47 & 0.09 & 0.023 & 0.009 & 0.006 & 0.003 \\
\hline
\end{tabular}

We further present the reference field intensity correlations as a function of frame time separation for the turbulent experiment case in Table 2.

Table 2. Turbulent Case: Reference Field Intensity Correlations as a function of time separation.

\begin{tabular}{lccccccc}
\hline$\Delta i$ & 0 & 1 & 2 & 3 & 5 & 10 & 20 \\
$R_{+\Delta i}$ & 1.0 & 0.41 & 0.12 & 0.03 & 0.009 & 0.005 & 0.006 \\
$R_{-\Delta i}$ & 1.0 & 0.41 & 0.12 & 0.03 & 0.009 & 0.005 & 0.006 \\
\hline
\end{tabular}

As with our results for the non-turbulent case Table 2 presents the details of the reference frame correlations for the experiment in the turbulence case. In Figure 8a,b a graph is overlaid on the $\Delta i=0$ ghost image that displays the track of the "ARL" as it moves across the field of view in the non-turbulent and turbulence case, respectively. The results shown in Figure 8a,b demonstrate that a time separation between the bucket and reference fields gives an apparent change in location of the ghost image even through strong laboratory turbulence. 

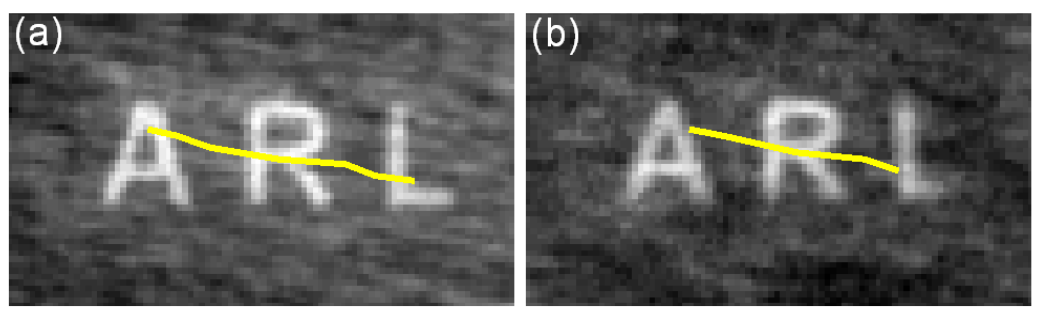

Figure 8. Lines overlayed on the time separation 0 ghost image "ARL" indicates the track that the ghost image follows based on the location of the middle of the letter " $R$ " for time separations $-20,-15,-10,0,+10,+15,+20$ (a) Non-turbulent conditions. (b) Turbulent conditions.

In addition to demonstrating the motion of ghost images due to the rotation of the ground glass additional information can be extracted from the time lagged joint measurements. For example, the letter " $\mathrm{R}$ " is reconstructed by extracting and compositing a new ghost image made up of columns of the ghost images at time separations as they translate past a line of pixels (Figure 9). The resolution of the new ghost imaging is a function of the speed of rotation of the ground glass. For example, a slower rotating ground glass will contribute to greater horizontal resolution of the ghost image. In this case we achieved higher resolution in Figure 9 than is available from any of the individual ghost images. A more detailed example of this is presented below with regard to imaging a U.S. Revolutionary War toy soldier.

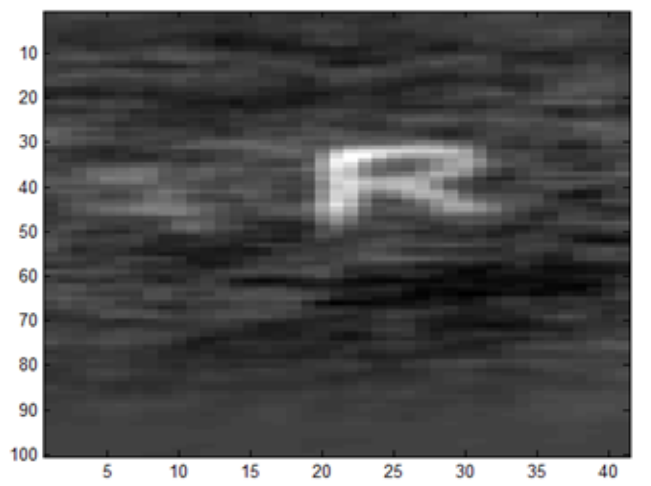

Figure 9. Sample of extracting a column of pixels at horizotonal position 60 of the ghost image as a function of the shifted frame index.

\subsection{Single Beam 2D Object Experiment Results}

The experimental setup to perform a second set of experiments is shown in Figure 10. It is also similar to previous ghost imaging setups $[1,2,15,16]$ except that a single illuminating beam was used, a faster charged coupled device (CCD) framing rate was implemented, and a slower rotation rate was used to resolve the time of rotation of the ground glass. We performed these experiments for successive high speed frames so as to track the laser illumination through rotating ground glass disturbance over the reference and target fields. The single beam space-time ghost imaging experiments consisted of using the reference field images at each time $t_{r e f}=t_{i}$ and a bucket field measured at a separate time $t_{b}=t_{i+\Delta i}$. This was performed for each time separation of $\Delta i=N_{1}$ to $N_{2}$ frames, where in some of our 
experiments $N_{1}=-500$ and $N_{2}=-545$. Interestingly, at coincident times, i.e., $\Delta i=0$, no space-time ghost images are possible in this configuration.

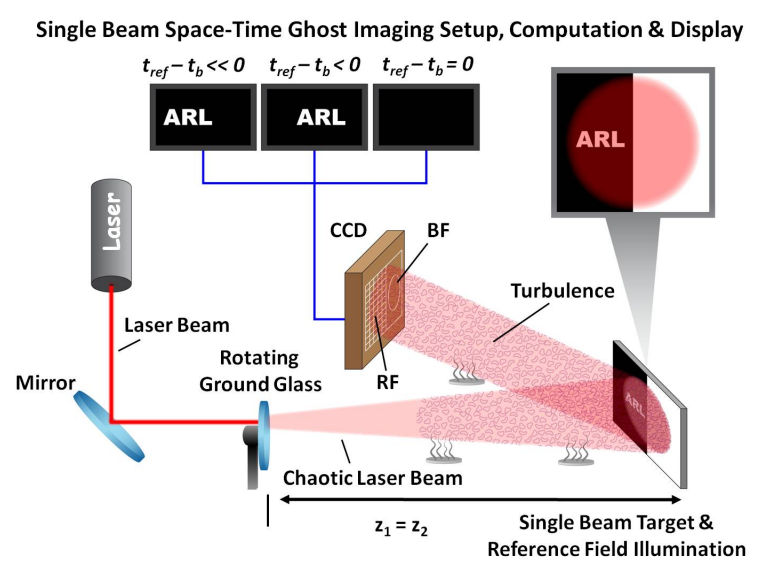

Figure 10. Layout for the single beam space-time ghost-imaging experiments. A CCD collected reference field (RF) and bucket field (BF) images at time $t_{i}$. The space-time "ARL" images at the top of the figure are representative results when the reference field measurements are correlated with coincident $\left(t_{r e f}-t_{b}=0\right)$, later $\left(t_{r e f}-t_{b}<0\right)$, or much later $\left(t_{r e f}-t_{b}<<0\right)$ bucket photon measurements.

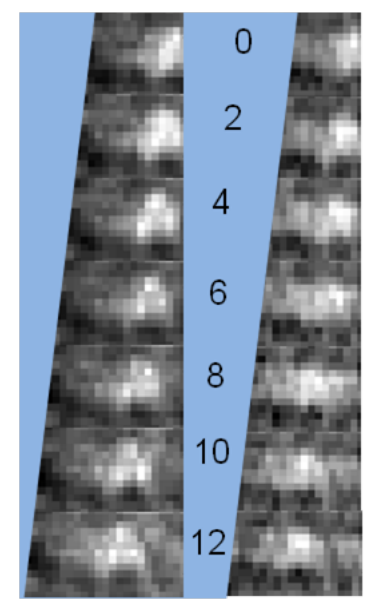

Figure 11. A series of single beam space-time ghost images. Left panel: Images were generated under conditions of no turbulence. Right panel: Images generated under conditions of strong laboratory turbulence. The ghost images are presented as a function of bucket measurement frame separation time relative to the reference frame measurement. Note that the "A" in the turbulence case is not well resolved. Ghost images are shown for up to 12 additional frames beyond the time separation of -524 frames.

Single beam space-time ghost imaging of the letter " $A$ " was performed first in non-turbulent conditions where the heating elements in Figure 10 were turned off. We found that each ghost image was translated according to the value of the time lag index $\Delta i$. When played as a movie the ghost image is seen to smoothly translate across the computer screen, the amount of translation being proportional to the time lag index. The translation appeared to be consistent with the light scattering variation due to the 
motion of the ground glass scattering centers. Images of these time lag ghost images in non-turbulent conditions are presented on the left side of Figure 11 and in Figure 12. While these space-time ghost images have many characteristics of our earlier time coincident ghost images, the generation of ghost images using a single illuminating beam where the reference and bucket measurements are not time coincident with each other is quite interesting.

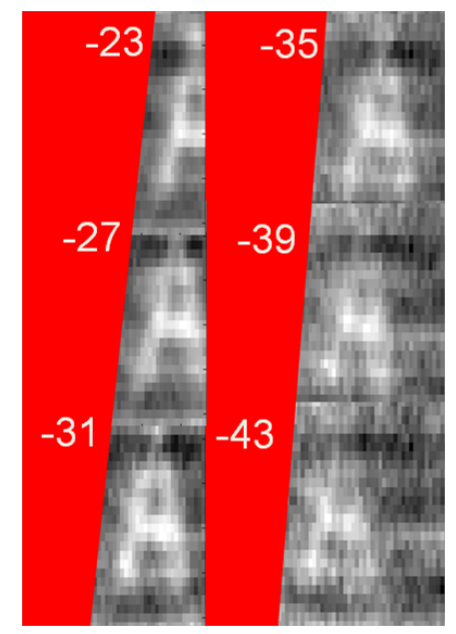

Figure 12. A series of single beam space-time ghost images was generated under conditions of no turbulence. The ghost images are presented as a function of bucket measurement frame separation time relative to the reference frame measurement. Ghost image results are shown for up to 43 additional frames beyond the separation of -501 frames.

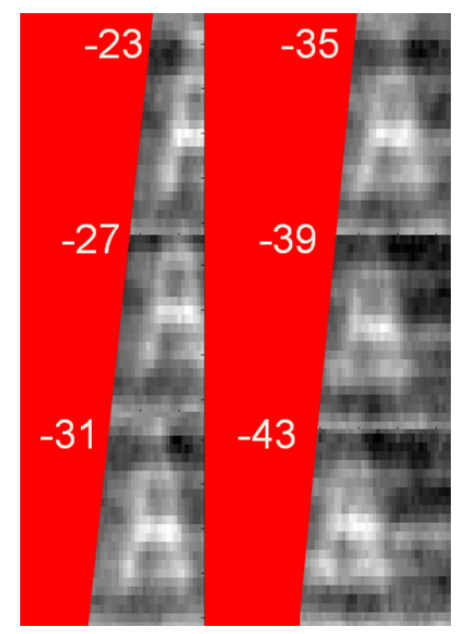

Figure 13. A series of single beam space-time ghost images was generated under conditions of high turbulence. The ghost images are presented as a function of bucket measurement frame separation time relative to the reference frame measurement. Note: Offset is -501 frames.

Single beam space-time ghost imaging experiments were then performed with turbulence, where the set of heating elements depicted in Figure 10 were turned on to $550{ }^{\circ} \mathrm{C}$ to generate strong laboratory turbulence. Images of the time lag ghost images generated with turbulence are presented on the right side of Figure 11 and in Figure 13. These results again demonstrate that a time separation between the 
bucket and reference fields gives an apparent change in location of the ghost image, even through strong laboratory turbulence. We note that the single beam space-time ghost images from the turbulence case were slightly degraded in comparison to the ghost images for the non-turbulent case.

\subsection{Single Beam 3D Object Experiment Results}

A 3D object was space-time ghost imaged with a single beam using the layout of Figure 10. The experiments imaged a U.S. Revolutionary War toy soldier. Figure 14 presents a single measurement of the toy soldier.

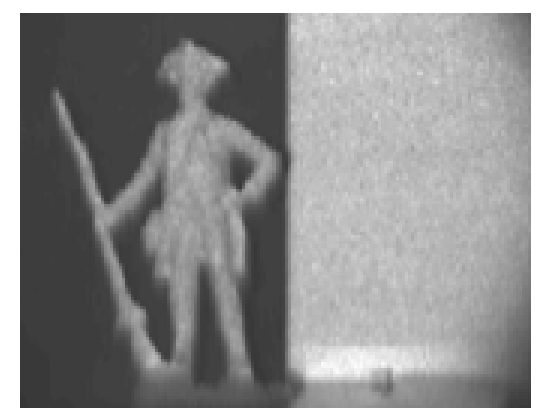

Figure 14. Single measurement of a 3D soldier model using the layout of Figure 10.

Measurements from these experiments were analyzed by extracting a single column of the ghost image generated for a particular time lag and stored in an image over all the time lags computed. Results from experiments performed when the ground glass rotation rate was $1^{\circ}$ per second are presented in Figure 15. The reference fields used in the ghost image calculations were $4 \times 4$ pixel spatial averages of the area shown in Figure 14 . Figure 15a is a composite ghost image generated from 180 time-delays and Figure $15 \mathrm{~b}$ is a sample of a single ghost image at a single time lag.
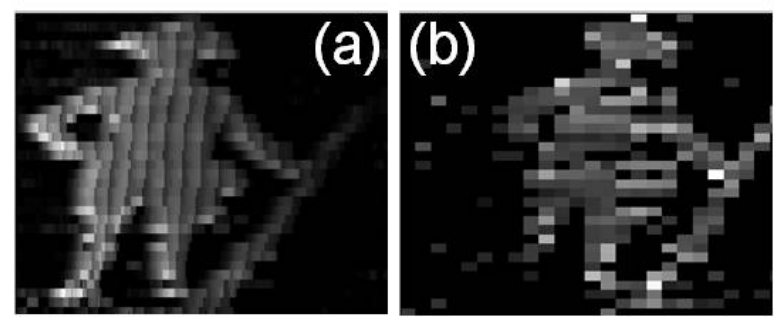

Figure 15. (a) Sample of extracting a column of pixels at horizotonal position of a ghost image of a 3D object as a function of the shifted frame index. (b) single ghost image at one $\Delta i$. The ground glass rotated at $1^{\circ}$ per second.

When the rotation speed of the ground glass was decreased to $\frac{1}{3}^{\circ}$ per second much more resolution and information of the target can be generated as is shown in Figure 16. The increased vertical resolution shown in Figure 16 is due to the use of $2 \times 2$ spatially averaged reference fields. The banding evident in Figures $15 \mathrm{a}$ and 16 is due to the time lagged reference fields correlation maxima moving between pixels. This in fact, provides sub-pixel scale information about the target. The banding has some contouring properties that helps illustrate $3 \mathrm{D}$ features of the object such as being in front of a plane. It 
should be noted that the correlations shown in Table 1 are from a multi sensor two-beam experiment and one should be cautious in applying those results to this multi sensor single beam experiment and that Equation (32) is derived for the two-photon interference phenomena where there is a small delay between the measurements. However the aspects relating to the ground glass induced phases apply also to other problems.

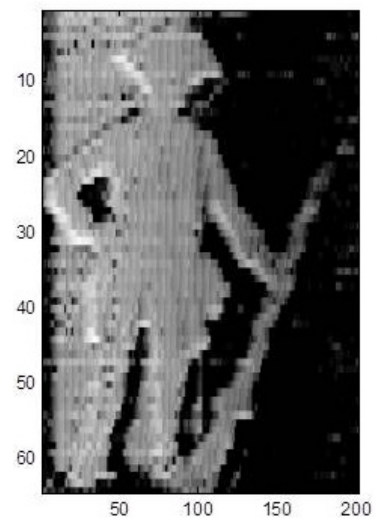

Figure 16. Sample of extracting a column of pixels at horizotonal position of a ghost image of a 3D object as a function of the shifted frame index in a proper aspect ratio. The ground glass rotated at $\frac{1}{3}^{\circ}$ per second.

For the cases illustrated in Figures 14-16 the soldier target model was approximately $3 \mathrm{~cm}$ tall and $2 \mathrm{~cm}$ wide from the elbow to the tip of the musket. The rotating ground glass was illuminated with a $633 \mathrm{~nm}$ wavelength laser. Each pixel of the camera $8 \mu \mathrm{m} \times 8 \mu \mathrm{m}$ and the target occupied an area of $3 \mathrm{~cm}$ vertical $\times 2 \mathrm{~cm}$ horizontal (125 pixels vertical $\times 100$ pixel horizontal) for $0.24 \mathrm{~mm}$ per pixel in the vertical and $0.2 \mathrm{~mm}$ per pixel in the horizontal. For Figure $15 \mathrm{a} 4 \times 4$ spatial averaging produced a composite pixels resolution of $0.96 \mathrm{~mm}$ by $0.8 \mathrm{~mm}$. Since 180 time shifts were needed to generate the composite ghost image at $13 \mathrm{fps}$ the 180 shifts at a rotation rate of $1^{\circ}$ per second, assuming $1 \mathrm{~ms}$ integration time, took $13.85 \mathrm{~s}$ to measure. Thus the horizontal speed of the illumination across the object is approximately $1.44 \mathrm{~mm} / \mathrm{s}$. With the rotation speed for Figure 16 being $\frac{1}{3}^{\circ}$ per second the translation of the illumination across the target took 3 times as long for a speed of approximately $0.48 \mathrm{~mm} / \mathrm{s}$.

\subsection{Virtual Storage of Ghost Images in Space-Time}

Our space-time ghost imaging approach has the ability to store more ghost images in the space-time dimensions than in just the space dimensions. For example, a single ghost image may be produced by 1000 frames of data. However, 40 or more shifted quality space-time ghost images may be produced with the same size data set using our setup, which raises the information content of each photon by more than an order of magnitude. It is likely that further improvements in our space-time imaging approach will raise the information content by even more orders of magnitude. Thus, we have demonstrated the creation of space-time ghost imaging, a new type of quantum image virtual memory in space-time, and the storage of much more information per photon measured. The moved ghost images are in fact new images that are stored in the dynamic memory in the same ghost imaging data sets thus producing more information per photon measured than was previously realized. 


\section{Theory for Space-Time Ghost Imaging}

A theory for space-time ghost imaging is developed below as an extension of conventional spatial ghost imaging. Space-time ghost imaging achieves ghost images with non-coincident measurements in addition to coincident measurements. Conventional spatial ghost imaging only uses coincident measurements at two different photo-sensors, a reference beam sensor and a photon bucket sensor. In thermal Ghost Imaging the beam splitter acts as a quantum operator on the photon quantum wavefunction. In quantum theory, a unitary operator relates the field operators at the input ports of the beamsplitter with the field operators at its output ports as a unitary transformation. The electromagnetic fields propagate from the source to the beamsplitter and then to the photo-detectors, following certain physical rules. With coincidence measurements we expect to be able to extract a ghost image. Ghost Imaging is the result of the turbulence-free point-to-point image-forming correlation, which is caused by two-photon interference: that is, superposition between paired two-photon amplitudes, corresponding to two different yet indistinguishable alternative ways of triggering a joint-detection event by two randomly distributed independent photons. An analysis illustrating the basic concepts follows. The analysis will be divided into three steps. First, we show that the point-to-point correlation between the object plane and the image plane is the result of two-photon interference. Second, we show that this correlation is turbulence-free. Third, we summarize the theory that effectively explains the results.

A joint-detection of two independent point photo-detectors measures the probability of observing a joint-detection event of two photons at space-time points $\left(\mathbf{r}_{1}, t_{1}\right)$ and $\left(\mathbf{r}_{2}, t_{2}\right)$, and is given by the Glauber's theory of photo-detection [20,21],

$$
\begin{aligned}
G^{(2)}\left(\mathbf{r}_{1}, t_{1} ; \mathbf{r}_{2}, t_{2}\right) & =\left\langle\left\langle E_{1}^{(-)} E_{2}^{(-)} E_{2}^{(+)} E_{1}^{(+)}\right\rangle_{\mathrm{QM}}\right\rangle_{\text {Ensemble }} \\
& =\operatorname{tr}\left\{\hat{\rho} E^{(-)}\left(\mathbf{r}_{1}, t_{1}\right) E^{(-)}\left(\mathbf{r}_{2}, t_{2}\right) E^{(+)}\left(\mathbf{r}_{2}, t_{2}\right) E^{(+)}\left(\mathbf{r}_{1}, t_{1}\right)\right\} .
\end{aligned}
$$

Here $\hat{\rho}$ is the density operator and the quantized thermal field, $E^{(-)}\left(\mathbf{r}_{j}, t_{j}\right)$ and $E^{(+)}\left(\mathbf{r}_{j}, t_{j}\right)$ are the negative and positive field operators at space-time coordinate $\left(\mathbf{r}_{j}, t_{j}\right)$. As is well known the coincidence counting rate of two photon counting detectors is proportional to $G^{(2)}\left(\mathbf{r}_{1}, t_{1} ; \mathbf{r}_{2}, t_{2}\right)$. To calculate the point-to-point correlation between the object plane and the image plane, we need (1) to estimate the state, or the density matrix, of the thermal radiation; and (2) to propagate the field operators from the radiation source to the object and the image planes. We will first estimate the state of thermal radiation at the single-photon level for photon counting measurements to explore the physics behind ghost imaging as two-photon interference. It is important to realize that in addition to timescales representing turbulence, sensor measurements, ground glass rotation and scattering, the lifetime of a radiative process must be considered and it is dependent on the Einstein A coefficient [22] which is fundamentally quantum in nature. Multiphoton ghost imaging experiments with different Einstein A coefficients will produce interference characteristic of those coefficients and therefore must be considered quantum. Thus, ghost imaging is fundamentally a quantum process and speckle theory does not readily account for such effects. A complete model will have all these space and time effects including the Einstein A coefficient.

A large transverse sized chaotic thermal source can be modeled as a large number of independent and randomly distributed point sub-sources $[1,2,15,16,23]$. Each point sub-source may in turn consist of a large number of independent atoms that are in their ground state, but some can be excited to a higher 
energy level $E_{2}$ and later return back to its ground state $E_{1}$. It is reasonable to assume that each atomic transition generates a field in the following single-photon state

$$
|\Psi\rangle \simeq|0\rangle+\epsilon \sum_{\mathbf{k}, s} f(\mathbf{k}, s) \hat{a}_{\mathbf{k}, s}^{\dagger}|0\rangle
$$

Here, $|\epsilon| \ll 1$ is the probability amplitude for the atomic transition. Since higher order terms representing multi-photon emissions have terms of $\epsilon^{2}, \epsilon^{3}, \ldots$ and that the magnitude of $\epsilon$ is small these terms fall off rapidly and can be safely neglected. The term $f(\mathbf{k}, s)=\left\langle\Psi_{\mathbf{k}, s} \mid \Psi\right\rangle$ is the probability amplitude for the radiation field to be in the single-photon state of wave number $\mathbf{k}$ and polarization $s$ : $\left|\Psi_{\mathbf{k}, s}\right\rangle=\left|1_{\mathbf{k}, s}\right\rangle=\hat{a}_{\mathbf{k}, s}^{\dagger}|0\rangle$. We assume a continuous distribution for $\mathbf{k}$ and one polarization for this simplified two-level system. The chaotic nature of the sub-radiations leads to a density operator that can be approximated as:

$$
\hat{\rho} \simeq|0\rangle\left\langle\left. 0|+| \epsilon\right|^{2} \int d \vec{\kappa} \hat{a}^{\dagger}(\vec{\kappa}) \mid 0\right\rangle\left\langle\left. 0|\hat{a}(\vec{\kappa})+| \epsilon\right|^{4} \int d \vec{\kappa} d \vec{\kappa}^{\prime} \hat{a}^{\dagger}(\vec{\kappa}) \hat{a}^{\dagger}\left(\vec{\kappa}^{\prime}\right) \mid 0\right\rangle\langle 0| \hat{a}\left(\vec{\kappa}^{\prime}\right) \hat{a}(\vec{\kappa}),
$$

where $\vec{\kappa}$ is the transverse wavevector. To simplify the calculation, we will focus on the transverse spatial correlation by assuming single-frequency transitions with monochromatic light as usual. Basically we are modeling the light source as an incoherent statistical mixture of single-photon states and two-photon states with equal probability of having any transverse momentum. The spatial part of the second-order coherence function is thus calculated as:

$$
G^{(2)}\left(\vec{\rho}_{1}, z_{1} ; \vec{\rho}_{2}, z_{2}\right) \simeq \int d \vec{\kappa} d \vec{\kappa}^{\prime}\left|\left\langle 0\left|E^{(+)}\left(\vec{\rho}_{2}, z_{2}\right) E^{(+)}\left(\vec{\rho}_{1}, z_{1}\right) \hat{a}^{\dagger}(\vec{\kappa}) \hat{a}^{\dagger}\left(\vec{\kappa}^{\prime}\right)\right| 0\right\rangle\right|^{2},
$$

where $\vec{\rho}_{j}$ is the transverse coordinate of the $j$ th photo-detector, $j=1,2$. The transverse part of the electric field operator can be written as:

$$
E^{(+)}\left(\vec{\rho}_{j}, z_{j}\right) \propto \int d \vec{\kappa} g_{j}\left(\vec{\rho}_{j}, z_{j} ; \vec{\kappa}\right) \hat{a}(\vec{\kappa})
$$

where $g_{j}\left(\vec{\rho}_{j}, z_{j} ; \vec{k}\right)$ is the Green's function, which propagates the field from the source to the photo-detector [24,25]. Substituting the field operators into Equation (9) we have

$$
G^{(2)}\left(\vec{\rho}_{1}, z_{1} ; \vec{\rho}_{2}, z_{2}\right)=\int d \vec{\kappa} d \vec{\kappa}^{\prime}\left|\frac{1}{\sqrt{2}}\left[g_{2}\left(\vec{\rho}_{2}, z_{2} ; \vec{\kappa}\right) g_{1}\left(\vec{\rho}_{1}, z_{1} ; \vec{\kappa}^{\prime}\right)+g_{2}\left(\vec{\rho}_{2}, z_{2} ; \vec{\kappa}^{\prime}\right) g_{1}\left(\vec{\rho}_{1}, z_{1} ; \vec{\kappa}\right)\right]\right|^{2}
$$

It is a key point that Equation (11) indicates interference between two quantum amplitudes, corresponding to two alternatives, different yet indistinguishable, which leads to a joint photo-detection event. This interference involves both arms of the optical setup as well as two distant photo-detection events at $\left(\vec{\rho}_{1}, z_{1}\right)$ and $\left(\vec{\rho}_{2}, z_{2}\right)$, respectively.

Figure 17 schematically illustrates the two alternatives for a pair of mode $\vec{\kappa}$ and $\vec{\kappa}^{\prime}$ to produce a joint photo-detection event. It is interesting to see that this superposition plays the same role as the symmetrized wavefunction of identical particles. The superposition of each pair of these amplitudes produces an individual interference in the joint-detection space of $\left(\vec{\rho}_{1}, z_{1}, t_{1} ; \vec{\rho}_{2}, z_{2}, t_{2}\right)$. A large number of these sub-interferences simply add together resulting in a point-to-point $G^{(2)}\left(\vec{\rho}_{1}, z_{1} ; \vec{\rho}_{2}, z_{2}\right)$ function. It is easy to see that each pair of the two-photon amplitudes illustrated in Figure 17 will superpose 
constructively whenever $D_{1}$ and $D_{2}$ are placed in the positions satisfying $\vec{\rho}_{1} \simeq \vec{\rho}_{2}$ and $z_{1} \simeq z_{2}$; and consequently, $G^{(2)}\left(\vec{\rho}_{1}, z_{1} ; \vec{\rho}_{2}, z_{2}\right)$ achieves its maximum value as the result of the sum of these individual constructive interferences. In other coordinates, however, the superposition of each individual pair of the two-photon amplitudes may yield different values between constructive maximum and destructive minimum due to unequal optical path propagation, resulting in an averaged background constant sum.

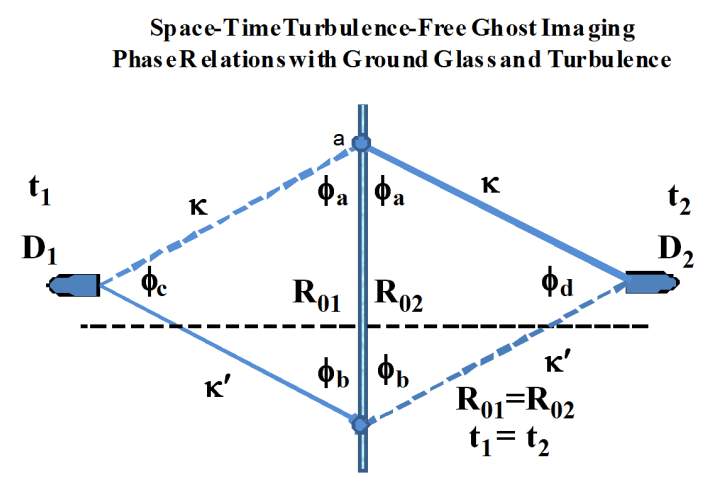

Figure 17. Schematic illustration of the two alternatives for a pair of mode $\vec{\kappa}$ and $\vec{\kappa}^{\prime}$ to produce a joint photo-detection event.

It is straightforward to verify the above interference picture mathematically. The Fresnel near field Green's function of free-propagation can be approximated as:

$$
\begin{aligned}
& g_{1}\left(\vec{\rho}_{1}, z_{1} ; \vec{\kappa}\right)=\int d \overrightarrow{\rho_{s}}\left\{\frac{-i \omega}{2 \pi c} \frac{e^{i \frac{\omega}{c} z_{1}}}{z_{1}} e^{i \frac{\omega}{2 c z_{1}}\left|\vec{\rho}_{1}-\vec{\rho}_{s}\right|^{2}}\right\} e^{-i \vec{\kappa} \cdot \overrightarrow{\rho_{s}}}, \\
& g_{2}\left(\vec{\rho}_{2}, z_{2} ; \vec{\kappa}\right)=\int d \overrightarrow{\rho_{s^{\prime}}}\left\{\frac{-i \omega}{2 \pi c} \frac{e^{i \frac{\omega}{c} z_{2}}}{z_{2}} e^{i \frac{\omega}{2 c z_{2}}\left|\overrightarrow{\rho_{2}}-\overrightarrow{\rho_{s^{\prime}}}\right|^{2}}\right\} e^{-i \vec{\kappa} \cdot \overrightarrow{\rho_{s^{\prime}}}},
\end{aligned}
$$

where $\overrightarrow{\rho_{s}}$ and $\overrightarrow{\rho_{s^{\prime}}}$ are transverse vectors in the source plane, and the field has propagated from the source to the $\vec{\rho}_{1}$ plane and $\vec{\rho}_{2}$ plane in arms 1 and 2, respectively. Substituting the Green's functions into Equation (11), the interference term (cross term of Equation (11)) turns to out be

$$
\left|\int d \vec{\rho}_{s} e^{i \frac{\omega}{c d}\left(\vec{\rho}_{1}-\vec{\rho}_{2}\right) \cdot \vec{\rho}_{s}}\right|^{2}=\operatorname{somb}^{2}\left[\frac{R}{d} \frac{\omega}{c}\left|\vec{\rho}_{1}-\vec{\rho}_{2}\right|\right]
$$

when choosing $z_{1}=z_{2}$. Where $\omega$ is the frequency of the electromagnetic radiation and $2 R$ is the diameter of the imaging lens, $d$ is the distance from the source to the detector, and $\frac{R}{d}$ is one half the angular size of illumination source as viewed from the detectors. The normalized second-order correlation function $g_{n-c o r}^{(2)}$ between the object plane and the image plane is therefore given by

$$
\begin{aligned}
g_{n-c o r}^{(2)}\left(\vec{\rho}_{1} ; \vec{\rho}_{2}\right) & \simeq 1+\operatorname{somb}^{2}\left[\frac{R}{d} \frac{\omega}{c}\left|\vec{\rho}_{1}-\vec{\rho}_{2}\right|\right] \\
& \simeq 1+\delta\left(\vec{\rho}_{n d 1}-\vec{\rho}_{n d 2}\right) .
\end{aligned}
$$

Note that $g_{n-c o r}^{(2)}$ is not the Green's function which is also typically written as $g$. It is to be realized that the argument of the somb function is nondimensional. The $\delta$ functions below are understood to be nondimensional where the arguments have been rescaled to allow them to be nondimensional and the normalized vector is $\vec{\rho}_{n d i}=\left(\frac{R}{d} \frac{\omega}{c}\right) \vec{\rho}_{i}$. From the Einstein, Podolsky and Rosen (EPR) [26] type nonlocal 
perspective, two photons that created a joint detection event of two distant photo-detectors have equal probabilities to be found at any coordinates on the object plane and the image plane, however, if one of them is observed at a certain position on the object (image) plane, the other one has twice chance to be observed in a unique position on the image (object) plane.

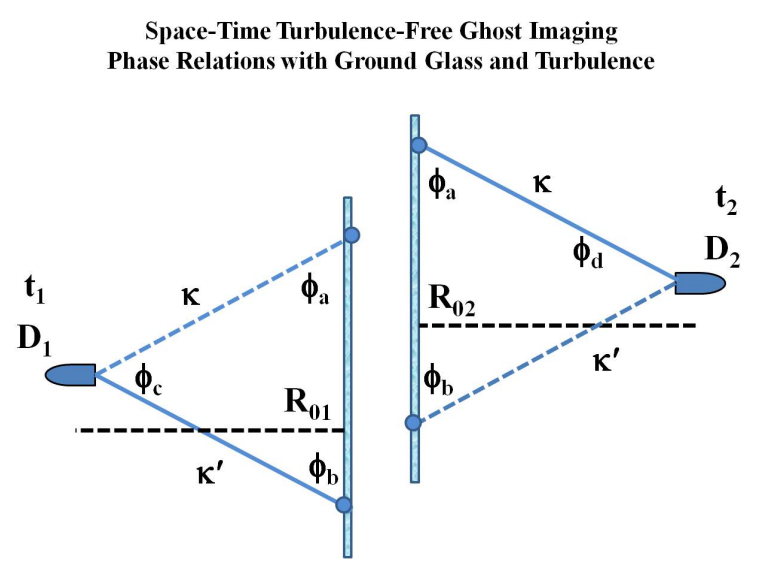

Figure 18. Two alternate but indistinguishable ways to trigger a photon detection at $D_{1}$ at time $t_{1}$ and a photon detection at $\mathrm{D}_{2}$ at time $\mathrm{t}_{2}$ from a rotating ground glass where one photon is emitted from subsource $a$ and the other photon is emitted from subsource $b$. For the joint detections to occur with the time separations the average phases at $\phi_{a}$ and $\phi_{b}$ must also translate as a function of time with the ground glass rotation. The vertical dashed line indicates the ground glass is translated vertically from position $R_{01}$ to $R_{02}$.

It is now necessary to introduce the ground glass effect directly into the space-time ghost imaging theory. Martienssen and Spiller [27] demonstrated that quickly rotating ground glass can convert a coherent laser beam into a chaotic radiation source suitable for optical experiments. Martienssen and Spiller characterized the time varying effect of ground glass on coherent radiation. In fact, ground glass is currently being used in fundamental experiments on studying the quantum superposition of coherent and pseudo-thermal light [11]. In the following we introduce a new model for ground glass that incorporates both time and space phase disturbance effects into the ghost imaging process. Starting from the $G^{(2)}$ equation we introduce phase disturbances that represent the effects of ground glass at each subsource position as measured at the detector points (see Figure 18). A relatively wide laser beam intersects a portion of the ground glass and light scatters off many disturbance elements on the ground glass. The scattering radiation contributions summed over the subsource elements and scattering elements interfere in the ghost imaging process. We expect that when the phase disturbances $\varphi_{A}\left(\vec{\rho}_{A}, \vec{\rho}_{2}, t_{2}, \vec{\kappa}\right)$, $\varphi_{A}\left(\vec{\rho}_{A}, \vec{\rho}_{1}, t_{1}, \vec{\kappa}\right), \varphi_{B}\left(\vec{\rho}_{B}, \vec{\rho}_{1}, t_{1}, \vec{\kappa}^{\prime}\right)$ and $\varphi_{B}\left(\vec{\rho}_{B}, \vec{\rho}_{2}, t_{2}, \vec{\kappa}^{\prime}\right)$ cancel in the probability amplitude formulation due to alternate and indistinguishable superpositions [28] at certain measurement space-time points, then the ghost image of the object can readily be seen in the imaging plane. A clear image cannot be formed at those space-time points where the phase disturbances do not cancel,

$$
G^{(2)}\left(\vec{\rho}_{1}, z_{1} ; \vec{\rho}_{2}, z_{2}\right)=\int d \vec{\kappa} d \vec{\kappa}^{\prime} \bullet \frac{1}{\sqrt{2}}\left[g_{2}\left(\vec{\rho}_{2}, z_{2} ; \vec{\kappa}^{\prime}\right) g_{1}\left(\vec{\rho}_{1}, z_{1} ; \vec{\kappa}\right)+g_{2}\left(\vec{\rho}_{2}, z_{2} ; \vec{\kappa}\right) g_{1}\left(\vec{\rho}_{1}, z_{1} ; \vec{\kappa}^{\prime}\right)\right]^{2}
$$

The cross term is

$$
G_{\text {crossterm }}^{(2)}=\int d \vec{\kappa} d \vec{\kappa}^{\prime} \bullet\left|\frac{1}{\sqrt{2}}\left[g_{2}\left(\vec{\rho}_{2}, z_{2} ; \vec{\kappa}^{\prime}\right) g_{1}\left(\vec{\rho}_{1}, z_{1} ; \vec{\kappa}\right) g_{2}^{*}\left(\vec{\rho}_{2}, z_{2} ; \vec{\kappa}\right) g_{1}^{*}\left(\vec{\rho}_{1}, z_{1} ; \vec{\kappa}^{\prime}\right)\right]\right|+\cdots
$$


Also,

$$
\begin{aligned}
& g_{1}\left(\overrightarrow{\rho_{1}}, z_{1} ; \vec{\kappa}\right)=\int d \overrightarrow{\rho_{s}} e^{i \frac{\omega}{2 c z_{1}}\left|\overrightarrow{\rho_{1}}-\overrightarrow{\rho_{s}}\right|^{2}} e^{-i \vec{\kappa} \cdot \overrightarrow{\rho_{s}}} \bullet e^{i \varphi_{A}\left(\overrightarrow{\rho_{s}}, t_{1}\right)} \\
& g_{2}\left(\vec{\rho}_{2}, z_{2} ; \vec{\kappa}^{\prime}\right)=\int d \overrightarrow{\rho_{s^{\prime}}} e^{i \frac{\omega}{2 c z_{2}}\left|\overrightarrow{\rho_{2}}-\overrightarrow{\rho_{s^{\prime}}}\right|^{2}} e^{-i \overrightarrow{\kappa^{\prime}} \cdot \overrightarrow{\rho_{s^{\prime}}}} \bullet e^{i \varphi_{B}\left(\overrightarrow{\rho_{s^{\prime}}}, t_{2}\right)} \\
& g_{1}^{*}\left(\vec{\rho}_{1}, z_{1} ; \vec{\kappa}^{\prime}\right)=\int d \overrightarrow{\rho_{s^{\prime}}} e^{-i \frac{\omega}{2 c z_{1}}\left|\overrightarrow{\rho_{1}}-\overrightarrow{\rho_{s^{\prime}}}\right|^{2}} e^{+i \overrightarrow{\kappa^{\prime} \cdot} \cdot \overrightarrow{\rho_{s^{\prime}}}} \bullet e^{-i \varphi_{B}\left(\overrightarrow{\rho_{s^{\prime}}}, t_{1}\right)} \\
& g_{2}^{*}\left(\overrightarrow{\rho_{2}}, z_{2} ; \vec{\kappa}\right)=\int d \overrightarrow{\rho_{s}} e^{-i \frac{\omega}{2 c z_{2}}\left|\overrightarrow{\rho_{2}}-\overrightarrow{\rho_{s}}\right|^{2}} e^{+i \vec{\kappa} \cdot \overrightarrow{\rho_{s}}} \bullet e^{-i \varphi_{A}\left(\overrightarrow{\rho_{s}}, t_{2}\right)}
\end{aligned}
$$

It is possible to integrate over $\vec{\kappa}$ and $\vec{\kappa}^{\prime}$ first to produce Dirac delta functions.

$$
\begin{aligned}
& g_{1}\left(\overrightarrow{\rho_{1}}, z_{1}\right) g_{2}^{*}\left(\overrightarrow{\rho_{2}}, z_{2}\right)=\int d \overrightarrow{\rho_{s^{\prime}}} \int d \overrightarrow{\rho_{s}} e^{i \frac{\omega}{2 c z_{1}}\left|\overrightarrow{\rho_{1}}-\overrightarrow{\rho_{s}}\right|^{2}} e^{-i \frac{\omega}{2 c z_{2}}\left|\overrightarrow{\rho_{2}}-\overrightarrow{\rho_{s^{\prime}}}\right|^{2}} \delta\left(\overrightarrow{\rho_{s}}-\overrightarrow{\rho_{s^{\prime}}}\right) \bullet e^{i \varphi_{A}\left(\overrightarrow{\rho_{s}}, t_{1}\right)} e^{-i \varphi_{A}\left(\overrightarrow{\rho_{s^{\prime}}}, t_{1}\right)} \\
& =\int d \overrightarrow{\rho_{s}} e^{i \frac{\omega}{2 c z_{1}}\left|\vec{\rho}_{1}-\vec{\rho}_{s}\right|^{2}} e^{-i \frac{\omega}{2 c z_{2}}\left|\vec{\rho}_{2}-\vec{\rho}_{s}\right|^{2}} \bullet e^{i \varphi_{A}\left(\overrightarrow{\rho_{s}}, t_{1}\right)} e^{-i \varphi_{A}\left(\overrightarrow{\rho_{s}}, t_{2}\right)} \\
& g_{2}\left(\overrightarrow{\rho_{2}}, z_{2}\right) g_{1}^{*}\left(\overrightarrow{\rho_{1}}, z_{1}\right)=\int d \overrightarrow{\rho_{s^{\prime}}} \int d \overrightarrow{\rho_{s}} e^{i \frac{\omega}{2 c z_{2}}\left|\overrightarrow{\rho_{2}}-\overrightarrow{\rho_{s^{\prime}}}\right|^{2}} e^{-i \frac{\omega}{2 c z_{1}}\left|\overrightarrow{\rho_{1}}-\overrightarrow{\rho_{s}}\right|^{2}} \delta\left(\overrightarrow{\rho_{s}}-\overrightarrow{\rho_{s^{\prime}}}\right) \bullet e^{-i \varphi_{B}\left(\overrightarrow{\rho_{s^{\prime}}}, t_{2}\right)} e^{+i \varphi_{B}\left(\overrightarrow{\rho_{s}}, t_{1}\right)} \\
& =\int d \overrightarrow{\rho_{s}} e^{i \frac{\omega}{2 c z_{2}}\left|\overrightarrow{\rho_{2}}-\overrightarrow{\rho_{s}}\right|^{2}} e^{-i \frac{\omega}{2 c z_{1}}\left|\overrightarrow{\rho_{1}}-\overrightarrow{\rho_{s}}\right|^{2}} \bullet e^{i \varphi_{B}\left(\overrightarrow{\rho_{s}}, t_{2}\right)} e^{-i \varphi_{B}\left(\overrightarrow{\rho_{s}}, t_{1}\right)} \\
& G_{\text {crossterm }}^{(2)}=g_{1}\left(\vec{\rho}_{1}, z_{1}\right) g_{2}^{*}\left(\vec{\rho}_{2}, z_{2}\right) g_{2}\left(\vec{\rho}_{2}, z_{2}\right) g_{1}^{*}\left(\vec{\rho}_{1}, z_{1}\right) \\
& =\int e^{i \frac{\omega}{2 c z_{1}}\left|\vec{\rho}_{1}-\vec{\rho}_{s}\right|^{2}} e^{-i \frac{\omega}{2 c z_{2}}\left|\vec{\rho}_{2}-\vec{\rho}_{s}\right|^{2}} e^{i \varphi_{A}\left(\vec{\rho}_{s}, t_{1}\right)-i \varphi_{A}\left(\vec{\rho}_{s}, t_{2}\right)} d \overrightarrow{\rho_{s}} \\
& \text { • } \int e^{i \frac{\omega}{2 c z_{1}}\left|\overrightarrow{\rho_{1}}-\overrightarrow{\rho_{s^{\prime}}}\right|^{2}} e^{-i \frac{\omega}{2 c z_{2}}\left|\overrightarrow{\rho_{2}}-\overrightarrow{\rho_{s^{\prime}}}\right|^{2}} e^{i \varphi_{B}\left(\overrightarrow{\rho_{s^{\prime}}}, t_{2}\right)-i \varphi_{B}\left(\overrightarrow{\rho_{s^{\prime}}}, t_{1}\right)} d \overrightarrow{\rho_{s^{\prime}}} \\
& G_{\text {crossterm }}^{(2)}=\int e^{i \frac{\omega}{c d}\left(\vec{\rho}_{1}-\vec{\rho}_{2}\right) \cdot \vec{\rho}_{s}} e^{i \varphi_{A}\left(\vec{\rho}_{s}, t_{1}\right)-i \varphi_{A}\left(\vec{\rho}_{s}, t_{2}\right)} d \overrightarrow{\rho_{s}} \bullet \int e^{-i \frac{\omega}{c d}\left(\vec{\rho}_{1}-\vec{\rho}_{2}\right) \cdot \vec{\rho}_{s^{\prime}}} e^{i \varphi_{B}\left(\vec{\rho}_{s^{\prime}}, t_{2}\right)-i \varphi_{B}\left(\vec{\rho}_{s^{\prime}}, t_{1}\right)} d \overrightarrow{\rho_{s^{\prime}}}
\end{aligned}
$$

But the phase disturbances

$$
e^{i \varphi_{s^{\prime}}\left(\vec{\rho}_{s^{\prime}}, t_{2}, \vec{\kappa}^{\prime}\right)}=\exp \left[i \varphi_{s^{\prime}}\left(\left(\vec{\rho}_{s 0^{\prime}}+\int_{t_{20}}^{t_{2}} \vec{v}\left(t^{\prime}\right) d t^{\prime}, t_{2}, \vec{\kappa}^{\prime}\right)\right)\right]
$$

and

$$
e^{i \varphi_{s}\left(\vec{\rho}_{s 0}, t_{1}, \vec{\kappa}\right)}=\exp \left[i \varphi_{s}\left(\vec{\rho}_{s}+\int_{t_{10}}^{t_{1}} \vec{v}\left(t^{\prime}\right) d t^{\prime}, t_{1}, \vec{\kappa}\right)\right]
$$

will influence the positions $\vec{\rho}_{1}=\vec{\rho}_{10}+\int_{t_{10}}^{t_{1}} \vec{v}\left(t^{\prime}\right) d t^{\prime}$ and $\vec{\rho}_{2}=\vec{\rho}_{20}+\int_{t_{20}}^{t_{2}} \vec{v}\left(t^{\prime}\right) d t^{\prime}$ due to the scattering disturbance at shifted time and locations due to the movement of the ground glass at velocity $\vec{v}$. Actually, the effect in the measurement plane is proportional to the velocity of the ground glass movement. On the ground glass at $\vec{\rho}_{s 0^{\prime}}$ a phase disturbance is related to the position $\vec{\rho}_{s^{\prime}}-\int_{t_{20}}^{t_{2}} \vec{v}\left(t^{\prime}\right) d t^{\prime}$ in the source plane so 
that $\vec{\rho}_{s 0^{\prime}}=\vec{\rho}_{s^{\prime}}-\int_{t_{20}}^{t_{2}} \vec{v}\left(t^{\prime}\right) d t^{\prime}$. Consequently, we substitute the position of the phase disturbance motions into the following and obtain

$$
\begin{aligned}
& g_{1}\left(\vec{\rho}_{1}, z_{1} ; \vec{\kappa}\right)=\int\left\{\frac{-i \omega}{2 \pi c} \frac{e^{i \frac{\omega}{c} z_{1}}}{z_{1}} \exp \left(i \frac{\omega}{2 c z_{1}}\left|\vec{\rho}_{10}-\int_{t_{10}}^{t_{1}} \vec{v}\left(t^{\prime}\right) d t^{\prime}-\overrightarrow{\rho_{s}}\right|^{2}\right)\right\} e^{-i \vec{\kappa} \cdot \overrightarrow{\rho_{s}}} \bullet e^{i \varphi_{s}\left(\overrightarrow{\rho_{s}}, t_{1}, \vec{\kappa}\right)} d \overrightarrow{\rho_{s}}, \quad(28) \\
& g_{2}\left(\vec{\rho}_{2}, z_{2} ; \vec{\kappa}^{\prime}\right)=\int\left\{\frac{-i \omega}{2 \pi c} \frac{e^{i \frac{\omega}{c} z_{2}}}{z_{2}} \exp \left(i \frac{\omega}{2 c z_{2}}\left|\vec{\rho}_{20}-\int_{t_{20}}^{t_{2}} \vec{v}\left(t^{\prime}\right) d t^{\prime}-\overrightarrow{\rho_{s^{\prime}}}\right|^{2}\right)\right\} e^{-i \vec{\kappa}^{\prime} \cdot \vec{\rho}_{s^{\prime}}} \bullet e^{i \varphi_{s^{\prime}}\left(\vec{\rho}_{s^{\prime}}, t_{2}, \vec{\kappa}^{\prime}\right)} d \overrightarrow{\rho_{s^{\prime}}},
\end{aligned}
$$

with an equivalent integral over the transverse vectors in the ground glass frame of reference.

Only at the translated positions can the phases $\varphi_{s}$ and $\varphi_{s^{\prime}}$ end up with a null result and interference creating a space-time ghost image occur. That is

$$
\vec{\rho}_{1}=\vec{\rho}_{10}-\int_{t_{10}}^{t_{1}} \vec{v}\left(t^{\prime}\right) d t^{\prime}
$$

and

$$
\vec{\rho}_{2}=\vec{\rho}_{20}+\int_{t_{20}}^{t_{2}} \vec{v}\left(t^{\prime}\right) d t^{\prime}
$$

The interference term (cross term) turns to out be

$$
\begin{aligned}
& \left|\int d \overrightarrow{\rho_{s}} e^{i \frac{\omega}{c d}\left(\overrightarrow{\rho_{1}}-\overrightarrow{\rho_{2}}\right) \cdot \overrightarrow{\rho_{s}}}\right|^{2}\left(\int e^{i \frac{\omega}{c d}\left(\overrightarrow{\rho_{1}}-\overrightarrow{\rho_{2}}\right) \cdot \overrightarrow{\rho_{s}}} e^{i \varphi_{A}\left(\overrightarrow{\rho_{s}}, t_{1}\right)-i \varphi_{A}\left(\overrightarrow{\rho_{s}}, t_{2}\right)} d \overrightarrow{\rho_{s}}\right) \bullet\left(\int e^{-i \frac{\omega}{c d}\left(\overrightarrow{\rho_{1}}-\overrightarrow{\rho_{2}}\right) \cdot \overrightarrow{\rho_{s^{\prime}}}} e^{i \varphi_{B}\left(\overrightarrow{\rho_{s^{\prime}}}, t_{1}\right)-i \varphi_{B}\left(\overrightarrow{\rho_{s^{\prime}}}, t_{2}\right)} d \overrightarrow{\rho_{s^{\prime}}}\right) \\
& =\operatorname{somb}^{2}\left[\frac{R}{d} \frac{\omega}{c}\left|\vec{\rho}_{10}-\vec{\rho}_{20}+\int_{t_{10}}^{t_{1}} \vec{v}\left(t^{\prime}\right) d t^{\prime}-\int_{t_{20}}^{t_{2}} \vec{v}\left(t^{\prime}\right) d t^{\prime}\right|\right]
\end{aligned}
$$

As an approximation

$$
\begin{aligned}
g_{n-c o r}^{(2)}\left(\vec{\rho}_{1} ; \vec{\rho}_{2}\right) & \simeq 1+\operatorname{somb}^{2}\left[\frac{R}{d} \frac{\omega}{c}\left|\vec{\rho}_{1}-\vec{\rho}_{2}\right|\right] \\
& \simeq 1+\delta\left(\vec{\rho}_{n 1}-\vec{\rho}_{n 2}\right) \\
& \simeq 1+\delta\left[\vec{\rho}_{n d 10}+\left(\int_{t_{10}}^{t_{1}} \vec{v}\left(t^{\prime}\right) d t^{\prime}\right)_{n d}-\vec{\rho}_{n d 20}-\left(\int_{t_{20}}^{t_{2}} \vec{v}_{n}\left(t^{\prime}\right) d t^{\prime}\right)_{n d}\right] \\
& \simeq 1+\delta\left[\vec{\rho}_{n d 10}-\vec{\rho}_{n d 20}+\left(\int_{t_{10}}^{t_{1}} \vec{v}\left(t^{\prime}\right) d t^{\prime}\right)_{n d}-\left(\int_{t_{20}}^{t_{2}} \vec{v}_{n}\left(t^{\prime}\right) d t^{\prime}\right)_{n d}\right] .
\end{aligned}
$$

Recall that the argument of the somb function is nondimensional. Similarly, the $\delta$ functions are understood to be nondimensional and the variables have been rescaled to allow them to be nondimensional,

$$
\begin{aligned}
\vec{\rho}_{n d i} & =\left(\frac{R}{d} \frac{\omega}{c}\right) \vec{\rho}_{i} \\
\left(\int_{t_{10}}^{t_{1}} \vec{v}\left(t^{\prime}\right) d t^{\prime}\right)_{n d} & =\left(\frac{R}{d} \frac{\omega}{c}\right) \int_{t_{10}}^{t_{1}} \vec{v}\left(t^{\prime}\right) d t^{\prime} \\
\left(\int_{t_{20}}^{t_{2}} \overrightarrow{v_{n}}\left(t^{\prime}\right) d t^{\prime}\right)_{n d} & \left.=\left(\frac{R}{d} \frac{\omega}{c}\right) \int_{t_{20}}^{t_{2}} \vec{v}\left(t^{\prime}\right) d t^{\prime}\right] .
\end{aligned}
$$


For a time period, the space-time ghost image is turbulence-free. Over long enough time delay as the time separation increases, we expect some spread of the distribution away from a Dirac delta function due to extension beyond the setup coherence time and the introduction of experimental error. We note that the two-photon model is the first to predict this effect of the ground glass with a turbulence-free characteristic. The non-classical character of the equation becomes evident when the somb function from two-photon second order interference approaches a non-factorizeable $\delta\left(\vec{\rho}_{n d 1}-\vec{\rho}_{n d 2}\right)$ function where the integration is over all possible subsources, Figure 19a, and the resolution exceeds classical first order resolution. A $4 \mathrm{f}$ speckle correlation model, Figure $19 \mathrm{~b}$ tends to produce factorizeable (classical) $\delta\left(\vec{\rho}_{n d 1}-\vec{\rho}_{n d s}\right) \cdot \delta\left(\vec{\rho}_{n d 2}-\vec{\rho}_{n d s^{\prime}}\right)$, where $\vec{\rho}_{n d s}$ is the transverse position of the source of the speckle. One has to integrate over classical measurements of $\vec{\rho}_{n d s}$ and $\vec{\rho}_{n d s^{\prime}}$ so the result tends to introduce classical speckle resolution. The resolution of these correlations is limited by the speckle size since speckle methods involve imaging the speckles. This is due to the speckle models using two classical imaging systems to image the source onto the image and object planes. Furthermore, we have experimentally generated ghost images with sensor photon counts of less than 10 above the background and there is no discernible speckle. Photon counts vary even in two slit interference experiments where photons traverse the slits one or more at a time. Physics does not tend to characterize these as speckle. So the use of speckle models to describe interference phenomena is problematic, whereas the use of multiphoton measurements has a sound characterization based on the fundamental photon measurement work of Glauber.

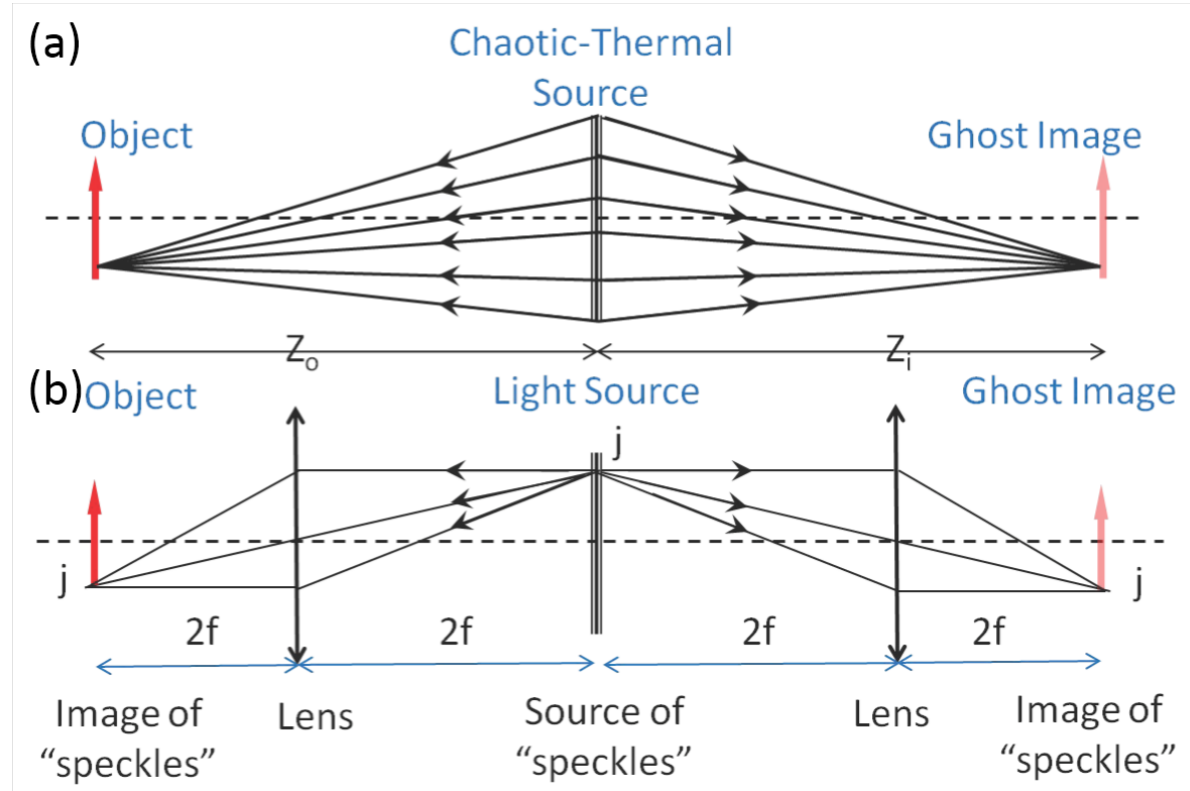

Figure 19. Comparison between the turbulence-free lensless ghost imaging experiments and speckle experiments. (a) Schematic setup of near field turbulence-free lensless ghost imaging experiments $[1,2,15,16]$. Each subsource of the source contributes to illuminating each point of the object and reference planes. Integrating over all the subsources produces a non-factorizeable $\delta\left(\vec{\rho}_{n d 1}-\vec{\rho}_{n d 2}\right)$ function. (b) Schematic setup of speckle experiments where the source of the speckle is imaged onto the object and to the reference plane. The correlation is a product of two first order delta functions, $\delta\left(\vec{\rho}_{n d 1}-\vec{\rho}_{n d s}\right) \cdot \delta\left(\vec{\rho}_{n d 2}-\vec{\rho}_{n d s}\right)$. 
The two-photon interference model was used because it correctly predicts turbulence-free ghost imaging while speckle models do not. Furthermore, our model incorporates phase information, produced in this case by a rotating ground glass, from two separate times. It is through the two-photon interference of these phases that leads to the generation of a clear image of the translated target.

The same two-photon interference model can be used for nonclassical light such as entangled photons. In fact it can be easily extended to other particles such as electrons and neutrons. The speckle model is not readily adaptable to such cases. While there are phase disturbances from turbulence and from ground glass the model predicts that the adverse effects of the phase disturbances from the turbulence is cancelled whereas the phase disturbances created by the ground glass actually enable the viewing of the object at certain time differences at certain positions where they tend to cancel. Of course, the phase disturbances can be characterized in terms of probability space-time moments and deviations. Thus, our space-time quantum imaging analysis allows quantitative insight into the movement of a phase object, such as ground glass, in the setup. Our approach uses fundamental physics based on the Glauber theory of photo-detection [20]. Glauber has shown that his model holds not only for the effects of individual photons but also for the effects of time averaged light and particularly he shows that quantum interference is observable from time averaged joint intensity measurements. Furthermore our two-photon interference model of two alternative and indistinguishable ways to generate a joint detection satisfies the Feynmann path integral formulation [28].

Why use a quantum model for space-time ghost imaging through turbulence rather than a classical speckle based model? First we recognize that all photons are quantum all of the time. When classical approximations are introduced inconsistencies may appear and care must be taken to not destroy or modify the information we are looking for. Using the quantum two-photon and multi-photon interference theory we successfully realized the first ghost image of a remote object and extending the quantum theory guided us to achieve turbulence-free ghost imaging beyond the capabilities of classical approaches. Thus we are in favor of this approach for future explorations as it is consistent with more experiments and introduces fewer errors into the physics formulation. For example, the multi-photon interference approach in ghost imaging allows for diversity in sensor and illumination location, timing, entanglement, size and sensitivity that is difficult to consider with classical approaches. As we all know, any theory claiming to comprehensively model the quantum nature of photons as a classical theory has thus far been shown to be inconsistent. Godel's second incompleteness theorem is often interpreted to mean that "an inconsistent model can prove everything including its own consistency." Our turbulence-free ghost imaging experiment cannot be correctly interpreted by a classical model or theory. Turbulence-free ghost imaging is a nonlocal phenomenon, which is the result of a nonlocal interference involving the superposition of two-photon amplitudes representing different alternative and indistinguishable ways for a pair of photons to produce a joint photodetection event at a distance. The insightful quantum theorist Feynmann recognized the importance of having a theory verified by experiments, "It doesn't matter how beautiful your theory is, it doesn't matter how smart you are. If it doesn't agree with experiment, it's wrong"'[29]. 


\section{Conclusions}

In conclusion we have demonstrated interesting features of space-time quantum imaging where the "reference" and "bucket" measurements need not be coincident in time to produce a ghost image of a remote object. The clearest individual time lagged space-time double beam quantum image is the ghost image with zero time lag, but the clarity of the images separated from this time lag is quite remarkable. Of further interest are the results displaying the "ARL" and letter "A" ghost image translation paths across the field of view. We believe that this effect is related to the motion of the light scattering features of the rotating ground glass and the physics of two-photon interference. A new model was developed for incorporating these space-time effects into ghost imaging. Our experiments demonstrate the potential of tracking moving objects with the ghost imaging approach. These features of ghost imaging may allow for greater adaptability and flexibility in the design and implementation of ghost imaging systems for many applications. In particular the coherence two-photon time and space scales can be explored with our ghost imaging experimental setup configuration. Furthermore, space-time quantum imaging has the potential to access orders of magnitude more information stored in the photon measurement data sets than was previously realized. In summary, the experiments provide a new testbed for exploring the fundamental physics of quantum imaging and suggest new pathways to explore quantum information storage and processing with multi-photon coincidence information and also in time delayed multi-photon interference. It is apparent that the space-time quantum imaging technology concepts and inventions can be implemented for many diverse applications to include practical ISR, quantum communications, and medical imaging applications with entangled or thermal photon illumination sources and detectors [30-32].

\section{Acknowledgments}

R.E. Meyers and K.S. Deacon thank the U.S. Army Research Laboratory for support. The authors would also like to thank A. Tunick, P. Hemmer, Y. Shih and S. Karmakar for helpful discussions.

\section{Author Contributions}

Both authors were fully involved in of the research: (1) conception and design, (2) drafting and revising, (3) final approval of the version to be published. The work progressed by a process of ongoing discussions and interactions between the authors. Both authors have read and approved the final manuscript.

\section{Appendix}

\section{A. Experiment Turbulence Characterization}

Atmospheric turbulence creates index of refraction variations that affect the paths of light propagation and provides a media for the quantum interference phenomena of quantum ghost imaging. The usual techniques for characterization of conventional optical turbulence are not generally well suited for the characterization of the effects of turbulence on quantum ghost imaging since they are based on first order 
interference and do not readily generalize to multiphoton interference. In the experiments in the lab and outdoors we want to document as much of the physics as possible to represent the turbulent conditions. In this appendix we characterize key aspects of the turbulence measurements used in the analysis of our laboratory ghost imaging experiments.

Turbulence is induced by instability mechanisms such as mechanical forcing and buoyancy and produce time-space variations in light propagation and photon counts, i.e., optical turbulence. While there is a world wide effort to measure and model more realistic turbulent atmospheric flows incorporating terrain, vegetation, heating, and cooling, common optical turbulence power law scalings are based on idealized assumptions of isotropy, homogeneity, and stationarity of the turbulence. In practice and by advanced Navier-Stokes model solutions [33], turbulence in the atmosphere [34] is never stationary, is generally inhomogeneous, has intermittency, and is anisotropic [35,36].

Turbulence is a result of strong stochastic space and time variations in the fluid properties such as velocity components $u_{i}(\mathbf{x}, t)$ and index of refraction $\eta(\mathbf{x}, t)$. Our experiments used realtime imaging, Figure A1, to extract properties for objective characterization of the anisotropic inhomogeneous turbulence. Figure A1 also shows the elongation and distortion of moving turbulent temperature fronts. Real-time imaging of the turbulence allowed us to investigate more detailed physics in the analysis of turbulence-free ghost imaging and its applications. Sequences of images of the laboratory optical turbulence were captured by an infrared camera.

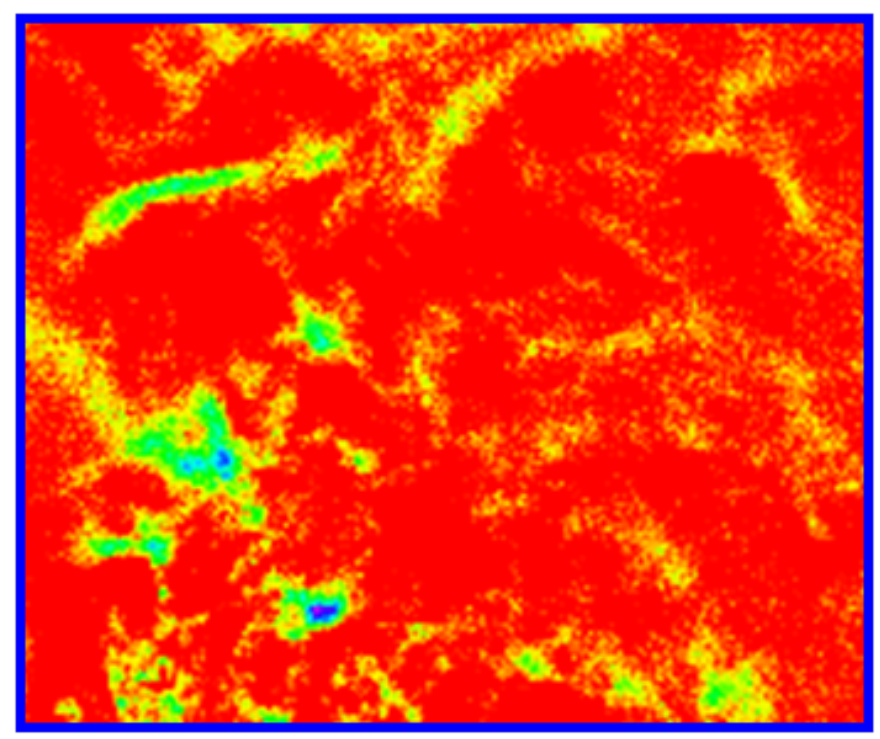

Figure A1. Typical Measured Real-Time Turbulence Caused by Displayed Heating Elements.

These frames were used to compute optical flow patterns [37] from which apparent turbulent velocities were derived. Velocity probability density functions (pdfs), velocity correlations, $\left\langle u_{i}\left(\mathbf{x}_{1}, t_{1}\right) u_{j}\left(\mathbf{x}_{2}, t_{2}\right)\right\rangle$, and their time and space scales were computed [35,36] (see Figures A2-A4). In the anisotropic, inhomogeneous, nonstationary turbulence a distribution of large and small scales were measured including velocity correlation space scales as small as 1-2.5 $\mathrm{mm}$ and turbulence velocity correlation time scales as small as $2.5-5 \mathrm{~ms}$. 
In convective turbulence there are larger magnitude vertical velocities with smaller magnitude negative velocities [36] as shown in Figure A2. Also typical of convective turbulence are short mean times for a velocity to go from negative to positive while the mean times for a velocity to go from positive to negative are larger [36] which is presented in Figure A3. The vertical velocity PDF shown in Figure A4 highlights the asymmetric and multi-modal characteristics typical of the turbulence for these experiments.

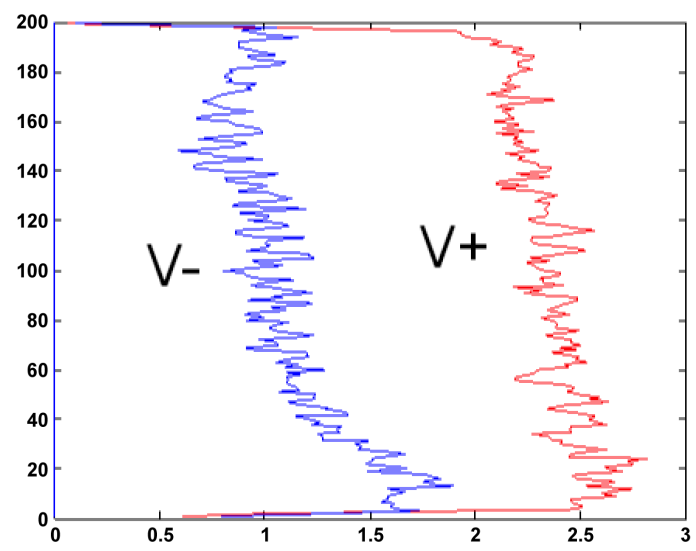

Figure A2. Expected Values of velocity upward (red) and downward (blue) as function of height in flow.

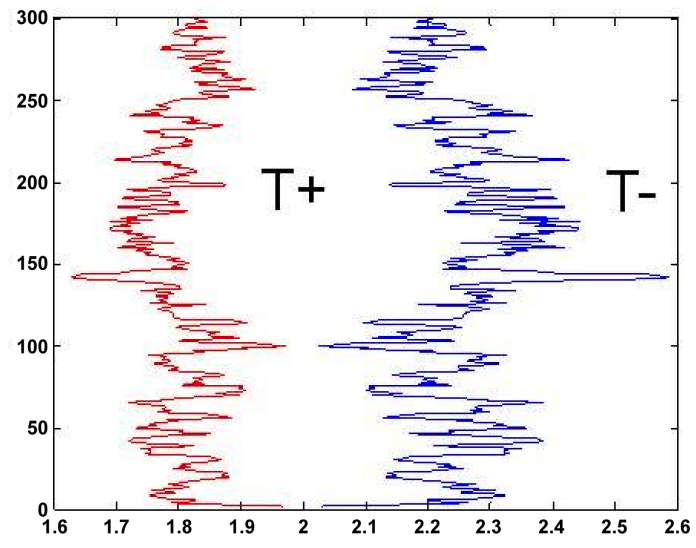

Figure A3. Map of time of reversal data $\mathrm{T}+, \mathrm{T}$ - as a function of height.

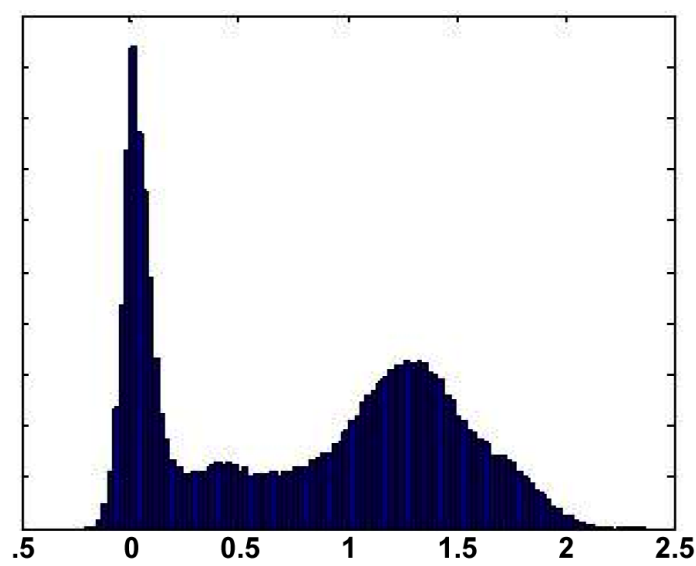

Figure A4. PDF of ensemble averaged vertical velocities. 
While each beam distorts and spreads due to turbulence, the point-to-point two-photon correlation between the image plane and the object plane is maintained. Small space turbulence scales indicate that the reference and bucket beams experience independent turbulence deviations, and small time correlations mean that image frames at different times also experience different turbulence realizations.

Optical turbulence is the variability of light propagating through $\eta(\mathbf{x}, t)$ fluctuations, and is often characterized by the structure parameter $\left\langle[\eta(\mathbf{x})-\eta(\mathbf{x}+r)]^{2}\right\rangle=C_{n}^{2} \mathcal{F}$ where $\mathcal{F}$ is a scaling function [38,39] that is often set to $r^{\frac{2}{3}} . \quad C_{n}^{2}$ is a standard means of characterizing both laboratory and atmospheric optical turbulence and has dimensions of lengt $h^{-\frac{2}{3}}$, rendering the structure function dimensionless. The values of $C_{n}^{2}$ is the experimental results shown here were in the range of $C_{n}^{2} \simeq 10^{-12}$ to $10^{-9}$ which is equivalent to extremely high levels of atmospheric turbulence [15]. A typical turbulence spectrum from our experiments is shown in Figure A5. The spectrum is the energy per wavenumber. A line overlayed on the measured spectrum indicating a $-\frac{5}{3}$ power law is based on near neutral turbulence stability conditions and simple models assuming isotropy, energy cascade, and dissipation. The $-\frac{17}{3}$ scaling, also overlayed, was developed from referenced optical communications atmospheric experiments [38,39]. The spectra, time of reversal maps, flow field imaging, and velocity pdf show that a large diversity of scales were present in the experiment turbulence flows. We can see that simple scaling laws are inadequate for all but idealized flows. Our data indicates that our multiphoton quantum imaging is turbulence-free even in strong anisotropic, inhomogeneous, intermittent, and nonstationary turbulence.

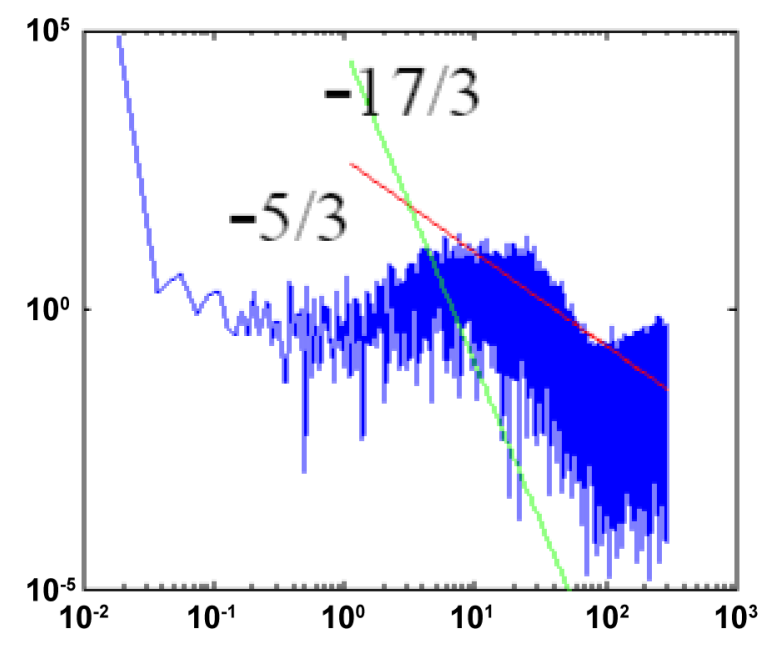

Figure A5. Typical turbulence spectrum.

\section{Conflicts of Interest}

The authors declare no conflict of interest.

\section{References}

1. Meyers, R.E.; Deacon, K.S.; Shih, Y.H. Ghost-imaging experiment by measuring reflected photons. Phys. Rev. A 2008, 77, doi:10.1103/PhysRevA.77.041801. 
2. Meyers, R.E.; Deacon, K.S.; Shih, Y.H. A new two-photon ghost imaging experiment with distortion study. J. Mod. Opt. 2007, 54, 2381-2392.

3. Dixon, P.B.; Howland, G.; Chan, K.W.C; O’Sullivan-Hale, C.; Brandon Rodenburg, B.; Hardey, N.D.; Shapiro, J.H.; Simon, D.D.; Sergienko, A.V.; Boyd, R.W.; et al. Quantum Ghost Imaging through Turbulence. Phys. Rev. A 2011, 83, doi:10.1103/PhysRevA.83.051803.

4. Brida, G.; Chekhova, M.V.; Fornaro, G.A.; Genovese, M.; Lopaeva, E.D.; Berchera, I.R. Systematic analysis of signal-to-noise ratio in bipartite ghost imaging with classical and quantum light. Phys. Rev. A 2011, 83, doi:10.1103/PhysRevA.83.063807.

5. Lopaeva, E.D.; Berchera, I.R.; Degiovanni, I.P.; Olivares, S.; Brida, G.; Genovese, M. Experimental realization of quantum illumination. Phys. Rev. Lett. 2013, 110, doi:10.1103/ PhysRevLett.110.153603.

6. Li, M.-F.; Zhang, Y.-R.; Luo, K.-H.; Wu, L.-A.; Fan, H. Time-correspondence differential ghost imaging. Phys. Rev. A 2013, 87, doi:10.1103/PhysRevA.87.033813.

7. Chen, H.; Peng, T.; Shih, Y.H. 100\% correlation of chaotic thermal light. Phys. Rev. A 2013, 88, doi:10.1103/PhysRevA.88.023808.

8. Meyers, R.E.; Deacon, K.S.; Tunick, A.D.; Shih, Y.H. Virtual ghost imaging through turbulence and obscurants using Bessel beam illumination. Appl. Phys. Lett. 2012, 100, doi:10.1063/1.3684613.

9. Katz, O.; Bromberg, Y.; Silberberg, Y. Ghost imaging with a single detector. Phys. Rev. A 2009, 79, doi:10.1103/PhysRevA.79.053840.

10. Shapiro, J.H. Computational ghost imaging. Phys. Rev. A 2008, 78, doi:10.1103/ PhysRevA.78.061802.

11. Liu, J; Zhou, Y.; Li, F.-L.; Xu, Z. The second-order interference between laser and thermal light. Europhys. Lett. 2014, 105, doi:10.1209/0295-5075/105/64007.

12. Shih, Y.H. The Physics of Ghost imaging. 2009, arXiv:0805.1166v5.

13. Peng, T.; Chen, H.; Shih, Y.H.; Scully, M.O. Delayed-choice quantum eraser with thermal light. Phys. Rev. Lett. 2014, 112, doi:10.1103/PhysRevLett.112.180401.

14. Scully, M.O. The time-dependent Schrodinger equation revisited: Quantum optical and classical Maxwell routes to Schrodinger's wave equation. In Time in Quantum Mechanics; Muga, G., Ruschhaupt, A., Campo, A., Eds.; Springer: Berlin/Heidelberg, Germany, 2010; Volume 2, pp. 15-24.

15. Meyers, R.E.; Deacon, K.S.; Shih, Y.H. Turbulence-free ghost imaging. Appl. Phys. Lett 2011, 98, doi:10.1063/1.3567931.

16. Meyers, R.E.; Deacon, K.S.; Shih, Y.H. Positive-negative turbulence-free ghost imaging. Appl. Phys. Lett. 2012, 100, doi:10.1063/1.3698158.

17. Meyers, R.E.; Deacon, K.S.; Tunick, A. Turbulence-free quantum ghost imaging experiments and results. In Proceedings of SPIE 8163, Quantum Communications and Quantum Imaging IX, San Diego, CA, USA, 21 August 2011.

18. Meyers, R.E.; Deacon, K.S.; Tunick, A. Turbulence-free space-time quantum imaging. In Proceedings of SPIE 8875, Quantum Communications and Quantum Imaging XI, San Diego, CA, USA, 25 August 2013. 
19. Meyers, R.E.; Deacon, K.S.; Tunick, A.D. Space-time quantum ghost imaging: Double beam and single beam. In Proceedings of SPIE 9225, Quantum Communications and Quantum Imaging XII, San Diego, CA, USA, 17 August 2014.

20. Glauber, R.J. The quantum theory of optical coherence. Phys. Rev. 1963, 130, 2529-2539.

21. Glauber, R.J. Coherent and incoherent states of the radiation field. Phys. Rev. 1963, 131, 2766-2788.

22. Einstein, A. On the quantum theory of radiation. In Sources of Quantum Mechanics; Dover Publications: New York, NY, USA, 1968.

23. Shih, Y.H. An Introduction to Quantum Optics: Photon and Biphoton Physics; CRC Press: Boca Raton, FL, USA, 2011.

24. Rubin, M.H. Transverse correlation in optical spontaneous parametric down-conversion. Phys. Rev. A 1996, 54, 5349-5360.

25. Goodman, J.W. Introduction to Fourier Optics; McGraw-Hill: New York, NY, USA, 1968.

26. Einstein, A.; Podolsky, B.; Rosen, N. Can quantum-mechanical description of physical reality be considered complete? Phys. Rev. 1935, 47, 777-780.

27. Martienssen, W; Spiller, E. Coherence and fluctuations in light beams. Am. J. Phys. 1964, 32, 919-926.

28. Feynmann, R.P.; Hibbs, A.R. Quantum Mechanics and Path Integrals; McGraw-Hill: New York, NY, USA, 1965.

29. Feynmann, R.P. The Best Mind Since Einstein; NOVA, PBS: Boston, MA, USA, 1993.

30. Meyers, R.E.; Deacon, K.S.; Tunick, A. Method and System for Producing Image Frames Using Quantum Properties. US Patent 8811763, 19 August 2014.

31. Meyers, R.E.; Deacon, K.S. Image Enhancement System and Method. US Patent 8811768, 19 August 2014.

32. Meyers, R.E.; Deacon, K.S. System and Method for Image Enhancement. US Patent 8532427, 10 September 2013.

33. Zak, M.; Zbilut, J.P.; Meyers, R.E. From Instability to Intelligence: Complexity and Predictability in Nonlinear Dynamics; Springer: Berlin/Heidelberg, Germany, 1997.

34. Sethuraman, S.; Meyers, R.E.; Brown, R.M. A comparison of a Eulerian and a Lagrangian time scale for over-water atmospheric flows during stable conditions. Bound.-Layer Meteorol. 1978, 14, 557-565.

35. Meyers, R.E.; O'Brien. E.E. The joint Pdf of a scalar and its gradient at a point in a turbulent fluid. Combust. Sci. Technol. 1981, 26, 123-134.

36. Meyers, R.E.; Ohmstede, W.D.; Cederwall, R.T. A model of finite velocity diffusion dynamics from large eddy simulation, exhibiting both space and time memory. In Proceedings of the 7th Symposium on Turbulence and Diffusion, Boulder, CO, USA, 12-15 November 1985.

37. Horn, B.K.P.; Schunck, B.G. Determining optical flow, In Proceedings of SPIE 0281, Techniques and Applications of Image Understanding, 319, Washington, D.C., USA, 21 April 1981.

38. Andrews, L.C.; Philips, R.L. Laser Beam Propagation through Random Media; SPIE Press: Bellingham, WA, USA, 2005. 
39. Tunick, A.D. Optical turbulence parameters characterized via optical measurements over a $2.33 \mathrm{~km}$ free-space laser path. Opt. Express 2008, 16, 14645-14654.

(C) 2015 by the authors; licensee MDPI, Basel, Switzerland. This article is an open access article distributed under the terms and conditions of the Creative Commons Attribution license (http://creativecommons.org/licenses/by/4.0/). 